\title{
Equivariant Cohomology over Lie Groupoids and Lie-Rinehart Algebras
}

\author{
JOHANNES HUEBSCHMANN \\ USTL, UFR de Mathématiques, CNRS-UMR 8524, 59655 Villeneuve d'Ascq Cedex, \\ France.e-mail: Johannes.Huebschmann@math.univ-lille1.fr
}

Received: 6 March 2009 / Revised: 31 July 2009 / Accepted: 4 August 2009

Published online: 27 October 2009 - (C) Springer 2009

\begin{abstract}
Using the language and terminology of relative homological algebra, in particular that of derived functors, we introduce equivariant cohomology over a general Lie-Rinehart algebra and equivariant de Rham cohomology over a locally trivial Lie groupoid in terms of suitably defined monads (also known as triples) and the associated standard constructions. This extends a characterization of equivariant de Rham cohomology in terms of derived functors developed earlier for the special case where the Lie groupoid is an ordinary Lie group, viewed as a Lie groupoid with a single object; in that theory over a Lie group, the ordinary Bott-Dupont-Shulman-Stasheff complex arises as an a posteriori object. We prove that, given a locally trivial Lie groupoid $\Omega$ and a smooth $\Omega$-manifold $f: M \rightarrow B_{\Omega}$ over the space $B_{\Omega}$ of objects of $\Omega$, the resulting $\Omega$-equivariant de Rham theory of $f$ reduces to the ordinary equivariant de Rham theory of a vertex manifold $f^{-1}(q)$ relative to the vertex group $\Omega_{q}^{q}$, for any vertex $q$ in the space $B_{\Omega}$ of objects of $\Omega$; this implies that the equivariant de Rham cohomology introduced here coincides with the stack de Rham cohomology of the associated transformation groupoid; thus this stack de Rham cohomology can be characterized as a relative derived functor. We introduce a notion of cone on a Lie-Rinehart algebra and in particular that of cone on a Lie algebroid. This cone is an indispensable tool for the description of the requisite monads.
\end{abstract}

Mathematics Subject Classification (2000). Primary 22A22, 55N91, 58H05;

Secondary 14F40, 17B65, 17B66, 18C15, 18G10, 22E65.

Keywords. Lie groupoid, Lie algebroid, Atiyah sequence, Lie-Rinehart algebra, differentiable cohomology, Lie-Rinehart cohomology, Borel construction, equivariant cohomology, equivariant de Rham cohomology relative to a groupoid, equivariant cohomology relative to a Lie-Rinehart algebra, monad and dual standard construction, comonad and standard construction, relative derived functor, cone on a Lie-Rinehart algebra, cone on a Lie algebroid.

\section{Contents}

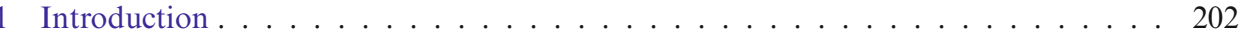

2 Monads and Relative Cohomology . . . . . . . . . . . . . . . . 207

3 Differentiable Cohomology over a Lie Groupoid . . . . . . . . . . . . . . . . 216

4 Comonads and Standard Constructions . . . . . . . . . . . . . . . . . 222 
5 Lie-Rinehart Equivariant Cohomology . . . . . . . . . . . . . . . . . 227

6 Extensions of Lie-Rinehart Algebras . . . . . . . . . . . . . . . 233

7 The Cone on the Lie Algebroid Associated with a Lie Groupoid . . . . . . . . 235

8 Equivariant de Rham Cohomology over a Lie Groupoid . . . . . . . . . . . . . . 238

9 Comparison with Other Notions of Lie Groupoid Cohomology . . . . . . . . . . 244

10 Outlook . . . . . . . . . . . . . . . . . . . . 247

\section{Introduction}

Equivariant de Rham cohomology over a Lie group is usually defined in terms of a certain bar-de Rham bicomplex; this bicomplex can be traced back, at least, to $[5,6,11,39,40]$ and serves, in de Rham theory, as a replacement for the Borel construction. In modern terminology, this bar-de Rham bicomplex yields the de Rham cohomology of the associated differentiable stack. However, the definition in terms of the bar-de Rham bicomplex hides the origins of equivariant cohomology, which is the theory of derived functors. Indeed the search for understanding the impact of symmetries encapsulated in a group on global invariants such as cohomology led to the cohomology of groups and thereafter to equivariant cohomology in general. Once the masters had isolated the cohomology of groups, they realized that the theory can be formalized in the language of derived functors. This led eventually to the description of derived functors in terms of (co)monads, also known as (co)triples, but we will exclusively use the monad-comonad terminology.

While the cohomology of groups is nowadays well understood as an instance of the theory of derived functors, it is less common to view equivariant cohomology as an instance of the theory of derived functors. However, given a topological group $G$ and a $G$-space $X$, over an arbitrary commutative ground ring $R$, the $G$-equivariant singular cohomology of $X$ can be characterized as the differential $\operatorname{Tor}^{C_{*}(G)}\left(R, C^{*}(X)\right)$. Here $C_{*}(G)$ refers to the differential graded $R$-algebra of singular chains on $G$ and $C^{*}(X)$ to the $R$-algebra of ordinary singular cochains on $X$, turned into a $C_{*}(G)$-module through the $G$-action on $X$. The standard object calculating this differential Tor is a bar construction; indeed, the Borel construction is well known to be the appropriate geometric analog of the corresponding bar construction. There is a perfectly reasonable (co)monad which leads to that differential Tor, and the appropriate comonad yields the homotopy quotient or Borel construction as an a posteriori object calculating equivariant cohomology. It is, perhaps, also worthwhile noting that, within the framework of relative homological algebra, the description of the $G$-equivariant cohomology of $X$ as the differential $\operatorname{Tor}^{C_{*}(G)}\left(R, C^{*}(X)\right)$ is self-explanatory and can be understood even without explicit reference to the theory of monads, etc. In [28], we have shown that homological perturbation theory, applied to that relative derived functor, yields a conceptual explanation for the phenomenon of Koszul duality.

In [29], we have introduced a monad which yields the equivariant de Rham cohomology with respect to a Lie group as a suitably defined relative derived 
Ext-functor, and we have also developed the corresponding infinitesimal theory. To capture global properties which rely on symmetries of a certain geometric situation that are more general than symmetries which can be encoded in a group, e.g., symmetries of a family of spaces rather than just of a space, in the present paper we start building similar equivariant theories for groupoids and Lie-Rinehart algebras in the framework of relative homological algebra.

The notions of injective module and injective resolution are fundamental. They have been developed by the masters to cope with situations where projective modules are not available. In contrast to the case of projective resolutions, the mechanism of injective resolutions is available in various situations where there are no projective objects; also the mechanism of injective resolutions can be adapted to take account of additional structure, e.g., that of differentiability of a module over a Lie group $G$; indeed, given a Lie group $G$, the appropriate way to resolve an object of the category of differentiable $G$-modules is by means of a differentiably injective resolution in the sense of [19].

The naive attempt to generalize equivariant cohomology over a Lie group to a theory over a general Lie groupoid is bound to fail: There is no obvious way to extend the bar-de Rham bicomplex to a complex over a Lie groupoid to arrive at the desired cohomology theory. Complications arise since the standard approach to a relative derived functor in terms of the nonhomogeneous resolution associated with the appropriate adjunction breaks down for groupoids. This difficulty resides in the fact that, as observed by K. Mackenzie in a remark on p. 285 of [32], for a general topological groupoid, there is no analog of the action used by Hochschild and Mostow on p. 369 of [19] which, for a Lie group $G$ and a vector space $V$, on the vector space $C^{\infty}(G, V)$ of smooth $V$-valued functions on $G$, is given by left (or right) translation. The putative extension of this kind of action would not be compatible with the variance constraint imposed by the groupoid action. See also Remark 3.1 below. Hence, for a general groupoid, the ordinary naive construction of a relatively injective module breaks down. The fact that, over a general locally trivial locally compact groupoid, any continuous module can be continuously embedded into a continuously injective one is thus not immediate; it has been established by K. Mackenzie in Theorem 1 of [32].

We will introduce equivariant cohomology over a general Lie-Rinehart algebra and equivariant de Rham theory over a locally trivial Lie groupoid in terms of suitably defined monads and the associated dual standard constructions. As for the terminology, we recall that, for purely formal or historical reasons, one refers to the standard construction associated with a comonad and to the dual standard construction associated with a monad. These monads extend the constructions in the predecessor [29] of the present paper but, for reasons explained above, we cannot simply extend the monad used in [29] to characterize ordinary equivariant de Rham cohomology over a Lie group and, indeed, in the present paper, we shall modify that monad so that the modified monad extends to Lie groupoids. We shall explain the details in Section 8 below. In Theorem 8.1, we shall then prove 
that, given the locally trivial Lie groupoid $\Omega$ over $B_{\Omega}$ and the left $\Omega$-manifold $f: M \rightarrow B_{\Omega}$ (fiber bundle endowed with a left $\Omega$-structure), for any object $q \in B_{\Omega}$, our $\Omega$-equivariant de Rham theory of $f$ is naturally isomorphic to the corresponding ordinary $\Omega_{q}^{q}$-equivariant de Rham cohomology of the corresponding fiber $f^{-1}(q)$ over $q$; here $\Omega_{q}^{q}$ refers to the vertex group at $q$. This is entirely consistent with Theorem 3 of [32] which says that, for a locally trivial topological groupoid, the rigid cohomology reduces to the Hochschild-Mostow theory for any vertex group with the corresponding coefficients; see also Proposition 3.6 below.

An indispensable tool in [29] is the cone $C \mathfrak{g}$ on an ordinary Lie algebra $\mathfrak{g}$, the cone being taken in the category of differential graded Lie algebras. In the present paper, given the Lie groupoid $\Omega$, we accordingly introduce the cone on the Lie algebroid $\lambda_{\Omega}$ or, equivalently, the corresponding cone on the corresponding LieRinehart algebra relative to the Atiyah sequence of the Lie groupoid; see Section 7 below. This extension is not entirely immediate since the naive construction of the cone on a Lie-Rinehart algebra, which we explore in Section 5, is not the correct notion of cone needed for equivariant de Rham cohomology relative to a Lie groupoid. The cone defined in terms of the Atiyah sequence is an indispensable tool for the description of the requisite monad defining the equivariant de Rham cohomology relative to a Lie groupoid.

Monads, comonads, standard constructions and dual standard constructions are explained in great detail in [35]. The idea of a monad goes back to [33] and that of a standard construction to [13], where the canonical flasque resolution of a sheaf is given as a suitable dual standard construction. Despite its flexibility and its vast range of possible applications, the theory of (co)monads, well known in category theory circles, has hardly been used elsewhere in mathematics except in algebraic topology where triples are quite common. Our approach to equivariant de Rham theory relative to a general locally trivial Lie groupoid clearly shows that monads and comonads deserve to be better known.

Here is a brief overview of the paper: In Section 2, we recall some of the basic notions of relative homological algebra used later in the paper, including monads and the associated dual standard constructions, and we illustrate these notions by means of certain examples involving groups and Lie groups; there examples are of fundamental importance later in the paper. In Section 3, we phrase the differentiable cohomology of locally trivial Lie groupoids in a language tailored to our purposes. In Section 4, we recall comonads and standard constructions, including the familiar comonadic description as a simplicial $G$-set or $G$-space of the universal object $E G$ associated with a Lie group $G$. Given the $G$-module $V$, we will then recall that the cosimplicial object $\operatorname{Map}(E G, V)$ coincides with the dual standard construction associated with the corresponding monad, cf. Proposition 4.1. In Section 5, we develop an equivariant theory relative to a Lie-Rinehart algebra. In Section 6, we explore extensions of Lie-Rinehart algebras and introduce a notion of cone relative to an extension of Lie-Rinehart algebras. In Section 7, we use the material developed before to introduce a notion of cone 
over the Lie algebroid associated with a Lie groupoid. In Section 8, we then introduce equivariant cohomology with respect to a locally trivial Lie groupoid as a relative derived functor by means of a suitable monad. In Section 9, we relate our approach to other notions of Lie groupoid cohomology in the literature. The term 'cohomology' may have several meanings; thus, rather than trying to develop the appropriate injective resolution, one can extend the construction of the standard complex defining smooth Lie group cohomology to the groupoid case; the resulting notion of cohomology has been explored in the literature, e.g., in [9] and [43]. This kind of Lie groupoid cohomology does not involve differential forms at all; it arises from application of the functor $C^{\infty}$ to the nerve of the groupoid, a construction substantially different from the one we use. While for smooth manifolds, ordinary real cohomology coincides with de Rham cohomology, under the present circumstances, the relationship is less clear. The naive attempt to recover the more direct definition which consists in applying the functor $C^{\infty}$ to the nerve is bound to fail since one would somehow try to compare apples and oranges. Indeed, one cannot directly apply the de Rham functor to the homotopy quotient arising from the nerve, and the de Rham cohomology of the associated stack is defined to be the total cohomology of the resulting cosimplicial de Rham complex. When the underlying Lie groupoid is actually an ordinary Lie group, viewed as a Lie groupoid with a single object, this cosimplicial de Rham complex reduces to the construction developed and explored by Bott et al. [5,6,11,40]. Prompted by a referee's report, we comment on the situation in Section 9. In particular, exploiting the appropriate Morita equivalence, we explain how our notion of equivariant cohomology over a locally trivial Lie groupoid includes a description of the corresponding stack de Rham cohomology of the associated transformation groupoid as a relative derived functor. Morita equivalence for the more direct definition is of course well known to hold, cf. e.g., [36]. Under the circumstances of the present paper, phrasing Morita equivalence is somewhat more delicate since it would have to involve the coefficients as well, and so far we do not understand Morita equivalence completely for the constructions developed here. The equivalence between our notion of equivariant cohomology and the corresponding stack de Rham cohomology of the associated transformation groupoid includes a version of Morita equivalence in a somewhat roundabout manner. Under the circumstances of the paper, the final form of Morita equivalence will presumably extend an old observation of Seda's [38] to the effect that, over a locally trivial topological groupoid, the functor given by restriction to a vertex group has the functor which assigns to a representation the associated induced fiber bundle as its left-adjoint; cf. also the proof of Proposition 3.6 below. In a final section we discuss various open questions.

The appropriate categorical setting for many constructions in the present paper is that of complete locally convex Hausdorff spaces, just as in [19] and [32]. We do not make this precise, to avoid an orgy of details related to topological vector spaces and leave the requisite details to the reader. We only mention here that, as 
on p. 376 of [19], given, e.g., the topological vector space $A$ and the Lie group $G$, we topologize $C^{\infty}(G, A)$ by taking for a fundamental system of neighborhoods of the origin of $C^{\infty}(G, A)$ the sets of the kind $N(C, D, U)$ where $C$ ranges over the compact subsets of $G$, the constituent $D$ over the finite sets of differential operators on $G$, the constituent $U$ over the neighborhoods of 0 in $A$, and $f$ being a member of $N(C, D, U)$ means that $\delta(f)(C) \subseteq U$ for every $\delta \in C$. In this manner we topologize, in particular, a space of the kind $C^{\infty}(G, \Gamma(\zeta))$ where $\zeta$ is a smooth vector bundle and $\Gamma(\zeta)$ ) its space of smooth sections, suitably topologized.

We will use the notation Ext and Tor for the corresponding infinite sequence of relative derived functors, not just for the first derived functors. This convention will be in force throughout this paper.

In equivariant de Rham theory, there is a certain dichotomy between left and right actions which creates some minor technical difficulties: In the standard formalism, when a Lie group $G$ acts on a smooth manifold $X$ from the left, the naturally induced $G$-action on the algebra of smooth functions on $X$ or, more generally, on the de Rham complex of $X$, is from the right, as is the induced infinitesimal action of the Lie algebra $\mathfrak{g}$ of $G$. As a side remark, we note that this fact has actually created some confusion in the literature. The formally appropriate approach to equivariant de Rham theory in terms of the monad technology involves right modules. This is the reason why, in [29], we built the theory systematically for right $G$-modules. However, when the Lie groupoid $\Omega$ with base manifold $B_{\Omega}$ acts on the fiber bundle $f: M \rightarrow B_{\Omega}$ from the left, the correct way to proceed is to build the theory in such a way that on the object corresponding to what was the algebra of functions before or, more generally the de Rham complex, the induced $\Omega$-action is still from the left. In terms of the earlier language of an action of a Lie group on a smooth manifold from the left, this simply means that we switch from the induced right action on the functions or on the de Rham complex to a left action in the standard way, an operation which is always possible since, for a group, the group algebra is a Hopf algebra in a canonical manner. This kind of switch is no longer possible over a general groupoid, though, and there is a single consistent procedure to build the theory, either left-handed or right-handed, and we proceed to built it left-handed; suffice it to mention that, had we decided to build the theory right-handed, we would in particular have to write differential operators on the right of the objects these operators apply to. In particular, right modules over a Lie-Rinehart algebra do not naively correspond to left modules; see [23] where the situation is discussed in detail. Piecing the various items correctly together and isolating the appropriate monads and categories is, then, a somewhat laborious puzzle.

In the literature, the $G$-equivariant cohomology $\mathrm{H}_{G}^{*}(\lambda)$ of a Lie algebroid $\lambda: E \rightarrow B$ acted upon by a compact Lie group $G$ has been explored, cf. e.g., [7]. This theory is substantially different from ours, though, that is a theory in which elements of a Lie groupoid and of a Lie algebroid are viewed as operators on a family of spaces that give rise to a corresponding equivariant theory, whereas in 
equivariant Lie algebroid cohomology the group $G$ acts on the Lie algebroid and hence on its associated differential graded algebra of forms.

I am indebted to the referees whose remarks made me clarify a number of issues and to K. Mackenzie for discussions related with his extension of the HochschildMostow cohomology theory for topological groups [19] to locally trivial topological groupoids and in particular with his construction of (relatively) injective modules over a locally trivial topological groupoid [32]. I owe a special debt of gratitude to Yvette Kosmann-Schwarzbach, to Jim Stasheff, and to Bertram Schwarzbach for a number of comments which helped improve the exposition.

\section{Monads and Relative Cohomology}

Before going into detail we note that we use the monad-comonad terminology exclusively rather than the equivalent terminology "triple", etc.

Let $\mathcal{M}$ be a category, $\mathcal{T}: \mathcal{M} \rightarrow \mathcal{M}$ an endofunctor, let $\mathcal{I}$ denote the identity functor of $\mathcal{M}$, and let $u: \mathcal{I} \rightarrow \mathcal{T}$ and $\mu: \mathcal{T}^{2} \longrightarrow \mathcal{T}$ be natural transformations. Recall that the triple $(\mathcal{T}, u, \mu)$ is said to be a monad over $\mathcal{M}$ provided that the following two diagrams commute:

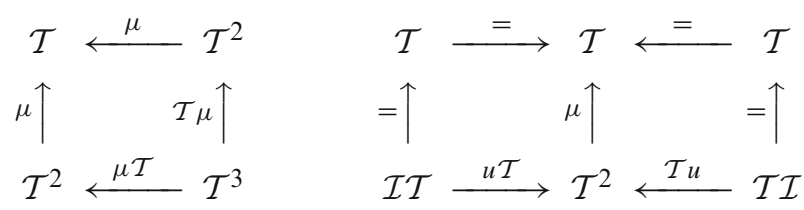

The natural transformations $u$ and $\mu$ are referred to as the unit and composition, respectively, of the monad.

The dual standard construction associated with the monad $(\mathcal{T}, u, \mu)$, cf. [12], [13] ("construction fondamentale" on p. 271), [35], yields the cosimplicial object

$$
\left(\mathcal{T}^{n+1}, \varepsilon^{j}: \mathcal{T}^{n+1} \rightarrow \mathcal{T}^{n+2}, \eta^{j}: \mathcal{T}^{n+2} \rightarrow \mathcal{T}^{n+1}\right)_{n \in \mathbb{N}} ;
$$

here, for $n \geq 0$,

$$
\begin{aligned}
& \varepsilon^{j}=\mathcal{T}^{j} u \mathcal{T}^{n-j+1}: \mathcal{T}^{n+1} \rightarrow \mathcal{T}^{n+2}, \quad j=0, \ldots, n+1, \\
& \eta^{j}=\mathcal{T}^{j} \mu \mathcal{T}^{n-j}: \mathcal{T}^{n+2} \rightarrow \mathcal{T}^{n+1}, \quad j=0, \ldots, n .
\end{aligned}
$$

Thus, given the object $V$ of $\mathcal{M}$, the system

$$
\mathbf{T}(V)=\left(\mathcal{T}^{n+1}(V), \varepsilon^{j}, \eta^{j}\right)_{n \in \mathbb{N}}
$$

is a cosimplicial object in $\mathcal{M}$; here we do not distinguish in notation between the natural transformations $\eta^{j}$ and $\varepsilon^{j}$ and the morphisms they induce after evaluation of the corresponding functors on an object. The dual notion of comonad will be reproduced in Section 4 below. 
An adjunction is well known to determine a monad (and a comonad) [35]: Let $\mathcal{D}$ and $\mathcal{M}$ be categories, let $\mathcal{G}: \mathcal{D} \rightarrow \mathcal{M}$ be a functor, suppose that the functor $\square: \mathcal{M} \rightarrow \mathcal{D}$ is left-adjoint to $\mathcal{G}$, and let

$$
\mathcal{T}=\mathcal{G} \square: \mathcal{M} \longrightarrow \mathcal{M} .
$$

Let $\mathcal{I}$ denote the identity functor, let $u: \mathcal{I} \rightarrow \mathcal{T}$ be the unit, $c: \square \mathcal{G} \rightarrow \mathcal{I}$ the counit of the adjunction, and let $\mu$ be the natural transformation

$$
\mu=\mathcal{G} c \square: \mathcal{G} \square \mathcal{G} \square=\mathcal{T}^{2} \longrightarrow \mathcal{T}=\mathcal{G} \square .
$$

The data $(\mathcal{T}, u, \mu)$ constitute a monad over the category $\mathcal{M}$. The associated dual standard construction then defines the relative $\operatorname{Ext}_{(\mathcal{M}, \mathcal{D})}$-functor, that is, the Extfunctor in the category $\mathcal{M}$ relative to the category $\mathcal{D}$.

EXAMPLE 2.1. Let $R$ be a commutative ring, $G$ a $\operatorname{group}, \operatorname{Mod}_{R}$ the category of $R$-modules, $\operatorname{Mod}_{R G}$ that of right $R G$-modules, let

$$
\mathcal{G}: \operatorname{Mod}_{R} \longrightarrow \operatorname{Mod}_{R G}
$$

be the familiar functor which assigns to the $R$-module $V$ the right $R G$-module $\operatorname{Map}(G, V)$, with right $G$-structure being given by left translation in $G$, and let

$$
\square: \operatorname{Mod}_{R G} \longrightarrow \operatorname{Mod}_{R}
$$

be the forgetful functor. The unit of the resulting adjunction is well known to be given by the assignment to the right $R G$-module $V$ of

$$
u_{V}: V \longrightarrow \operatorname{Map}(G, \square V), \quad v \longmapsto u_{v}: G \rightarrow \square V, \quad u_{v}(x)=v x, \quad v \in V, \quad x \in G .
$$

Likewise it is a standard fact that the counit of the adjunction is given by the assignment to the $R$-module $W$ of

$c_{W}: \square \operatorname{Map}(G, W) \longrightarrow W, \quad h \longmapsto h(e), \quad h: G \rightarrow W$.

Consequently the natural transformation

$\mu: \mathcal{T}^{2} \longrightarrow \mathcal{T}$

is given by the assignment to a right $R G$-module $V$ of the association

$$
\mu_{V}: \operatorname{Map}(G, \square \operatorname{Map}(G, \square V)) \longrightarrow \operatorname{Map}(G, \square V), \quad\left(\mu_{V}(\Phi)\right)(x)=\Phi(x)(e), \quad x \in G,
$$

where $\Phi$ ranges over maps of the kind $G \rightarrow \operatorname{Map}(G, \square V)$. Given the right $R G$-module $V$, the dual standard construction associated with $V$ and the resulting monad $(\mathcal{T}, u, \mu)$ yields the cosimplicial object

$$
\mathbf{T}(V)=\left(\operatorname{Map}\left(G^{\times(n+1)}, \square V\right), \varepsilon^{j}, \eta^{j}\right)_{n \in \mathbb{N}}
$$


in the category of right $R G$-modules, and the associated chain complex $|\mathbf{T}(V)|$, together with the injection $u_{V}$, is an injective resolution of $V$ in the category of right $R G$-modules. Given the right $R G$-module $W$, the chain complex $\operatorname{Hom}_{G}(|\mathbf{T}(V)|, W)$ then defines $\operatorname{Ext}_{G}(V, W)$. For intelligibility, we recall that the right $R G$-module structure on the degree $n$ constituent $\operatorname{Map}\left(G^{\times(n+1)}, \square V\right)$ is given by the association

$$
\begin{aligned}
& \operatorname{Map}\left(G^{\times(n+1)}, \square V\right) \times G \longrightarrow \operatorname{Map}\left(G^{\times(n+1)}, \square V\right),(\alpha, x) \mapsto \alpha \cdot x, \\
& (\alpha \cdot x)\left(x_{0}, x_{1}, \ldots, x_{n}\right)=\alpha\left(x x_{0}, x_{1}, \ldots, x_{n}\right),
\end{aligned}
$$

where $\alpha \in \operatorname{Map}\left(G^{\times(n+1)}, \square V\right)$ and $x, x_{0}, \ldots, x_{n} \in G$. All this is entirely standard and classical.

EXAMPLE 2.2. This example is a variant of Example 2.1, for Lie groups rather than just discrete groups and smooth maps rather than just set maps: Let the ground ring be that of the reals, $\mathbb{R}$, and let $G$ be a Lie group. We will use the notion of differentiable $G$-module in the sense of [19], and we will interpret the notation "Map" in the previous example as $C^{\infty}$, that is, as ordinary smooth maps. Let $\mathcal{D}=$ Vect, the category of real vector spaces, $\mathcal{M}=\operatorname{Mod}_{G}$ that of differentiable right $G$-modules, and let $\mathcal{G}_{G}: \operatorname{Vect} \rightarrow \operatorname{Mod}_{G}$ be the functor which assigns to the real vector space $V$ the $G$-representation

$$
\mathcal{G}_{G} V=C^{\infty}(G, V),
$$

endowed with the right $G$-module structure coming from left translation in $G$. We use the font $\mathcal{G}$ merely for convenience since this font is reminiscent of the notation $G$ in [35] for this kind of functor; this usage of the font $\mathcal{G}$ has nothing to do with our usage of the notation $G$ for the group variable. The functor $\mathcal{G}_{G}$ is right adjoint to the forgetful functor $\square: \operatorname{Mod}_{G} \rightarrow$ Vect and hence defines a monad $(\mathcal{T}, u, \mu)$ over the category $\operatorname{Mod}_{G}$. Given the differentiable right $G$-module $V$, the dual standard construction

$$
\mathbf{T}(V)=\left(C^{\infty}\left(G^{\times(n+1)}, \square V\right), \varepsilon^{j}, \eta^{j}\right)_{n \in \mathbb{N}}
$$

associated with $V$ and the monad $(\mathcal{T}, u, \mu)$ is a cosimplicial object in the category of differentiable right $G$-modules, and the associated chain complex $|\mathbf{T}(V)|$, together with the injection $u_{V}$, is a differentiably injective resolution of $V$ in the category of differentiable right $G$-modules; indeed, apart from the fact that we deal with right $G$-modules rather than left $G$-modules, this resolution is exactly one of the kind considered on p. 369 of [19]. Given the differentiable right $R G$-module $W$, the chain complex $\operatorname{Hom}_{G}(|\mathbf{T}(V)|, W)$ then defines the differentiable

$$
\operatorname{Ext}_{G}(V, W)=\operatorname{Ext}_{(\mathcal{M}, \mathcal{D})}(V, W),
$$


that is, the Ext in the category of differentiable right $G$-modules relative to the category of ordinary real vector spaces. The resolution $|\mathbf{T}(V)|$ of $V$ defines, in particular, the differentiable cohomology of $G$ with values in $V$ in the sense of [19], and we refer to that paper for details.

EXAMPLE 2.3. To prepare for our ultimate goal to develop an equivariant cohomology theory with respect to a Lie groupoid, we will now give an alternate description of the monad in Example 2.1. Thus, let $R$ be an arbitrary commutative ground ring, let $\mathcal{U}: \operatorname{Mod}_{R G} \longrightarrow \operatorname{Mod}_{R G}$ be the functor which assigns to the right $R G$-module $V$ the right $R G$-module $\mathcal{U}(V)=\operatorname{Map}(G, V)$, endowed with the right diagonal action

$$
\operatorname{Map}(G, V) \times G \longrightarrow \operatorname{Map}(G, V), \quad(\rho, x) \longmapsto \rho \cdot x
$$

given by

$$
(\rho \cdot x)(y)=(\rho(x y)) x, \quad x, y \in G, \quad \rho: G \rightarrow V,
$$

let $\omega$ be the natural transformation given by the assignment to the right $R G$-module $V$ of

$$
\omega=\omega_{V}: V \longrightarrow \operatorname{Map}(G, V), \quad v \longmapsto \omega_{v}: G \rightarrow V, \quad \omega_{v}(x)=v, \quad v \in V, \quad x \in G,
$$

and let

$$
v: \mathcal{U}^{2} \longrightarrow \mathcal{U}
$$

be the natural transformation given by the assignment to a right $R G$-module $V$ of the association

$$
\nu_{V}: \operatorname{Map}(G, \operatorname{Map}(G, V)) \longrightarrow \operatorname{Map}(G, V), \quad\left(\nu_{V}(\Psi)\right)(x)=\Psi(x)(x), \quad x \in G,
$$

where $\Psi$ ranges over maps of the kind $G \rightarrow \operatorname{Map}(G, V)$. The system $(\mathcal{U}, \omega, v)$ is a monad over the category $\operatorname{Mod}_{R G}$; it is, indeed, an alternate description of the monad $(\mathcal{T}, u, \mu)$ in Example 2.1, as we will show shortly. The following is well known and classical.

PROPOSITION 2.4. Let $V$ be a right $G$-module. Relative to the right G-module structures on $\operatorname{Map}(G, V)$ and $\operatorname{Map}(G, \square V)$, the map

$$
\vartheta=\vartheta_{V}: \operatorname{Map}(G, V) \longrightarrow \operatorname{Map}(G, \square V)
$$

given by

$$
\left(\vartheta_{V}(\rho)\right)(y)=(\rho(y)) y
$$


is a natural isomorphism of right $G$-modules, and

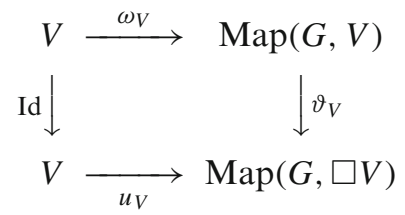

is a commutative diagram in the category of right $G$-modules. Furthermore, the diagram

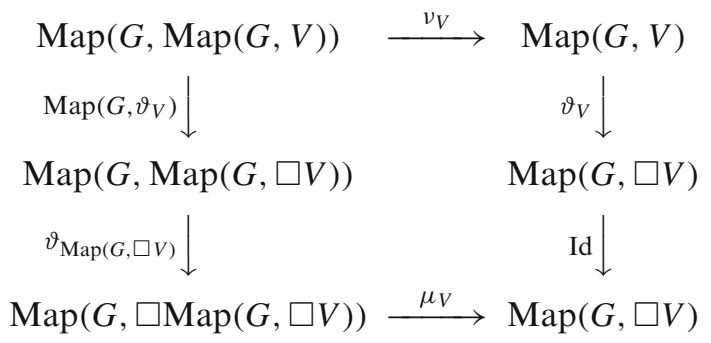

is commutative.

Proof. Indeed, given $y \in G$ and $\rho \in \operatorname{Map}(G, V)$,

$$
\left(\vartheta_{V} \rho\right)(y)=\left(\left(\vartheta_{V} \rho\right) \cdot y\right)(e)=\left(\vartheta_{V}(\rho \cdot y)\right)(e)=(\rho \cdot y)(e)=(\rho(y)) y .
$$

Consequently the diagram (2.8) is commutative. Furthermore, given $\Psi: G \rightarrow \operatorname{Map}(G, V)$ and $x \in G$,

$$
\begin{aligned}
\left(\vartheta_{V}\left(\nu_{V} \Psi\right)\right)(x) & =((\Psi(x))(x)) x \\
\left(\mu_{V}\left(\vartheta_{\operatorname{Map}(G, \square V)}\left(\operatorname{Map}\left(G, \vartheta_{V}\right)\right)(\Psi)\right)\right)(x) & =\left(\vartheta_{\operatorname{Map}(G, \square V)}\left(\operatorname{Map}\left(G, \vartheta_{V}\right)\right)(\Psi)\right)(x)(e)= \\
& =\left(\operatorname{Map}\left(G, \vartheta_{V}\right)(\Psi)\right)(x)(x)= \\
& =\left(\vartheta_{V}(\Psi(x))\right)(x)= \\
& =((\Psi(x))(x)) x
\end{aligned}
$$

whence the diagram (2.9) is commutative.

Remark 2.5. The isomorphism $\vartheta_{V}$ is determined by the requirement

$$
\left(\vartheta_{V} \rho\right)(e)=\rho(e), \quad \rho \in \operatorname{Map}(G, V) .
$$

Furthermore, on both $\operatorname{Map}(G, V)$ and $\operatorname{Map}(G, \square V)$ the right $G$-module structures relative to the copy of $G$ come from left translation in $G$.

COROLLARY 2.6. The natural transformation $\vartheta$ in Proposition 2.4 yields a natural isomorphism of monads from the monad $(\mathcal{U}, \omega, v)$ to the monad $(\mathcal{T}, u, \mu)$ in Example 2.1. 
Remark 2.7. This isomorphism between the two monads is presumably folk-lore and relies on the fact, well understood in the classical literature, cf. e.g., p. 212 of [17], that the diagonal map of the group $G$ turns the group $\operatorname{ring} R G$ of $G$ into a Hopf algebra.

Let $V$ be a right $R G$-module. The dual standard construction

$$
\mathbf{U}(V)=\left(\operatorname{Map}\left(G^{\times(n+1)}, V\right), \varepsilon^{j}, \eta^{j}\right)_{n \in \mathbb{N}}
$$

associated with $V$ and the monad $(\mathcal{U}, \omega, v)$ is a cosimplicial object in the category of right $R G$-modules. For intelligibility, we recall the cosimplicial structure; on the degree $n$ constituent $\operatorname{Map}\left(G^{\times(n+1)}, V\right)$, this structure is given by the familiar formulas

$$
\begin{aligned}
& \left(\varepsilon^{j}(\varphi)\right)\left(x_{0}, \ldots, x_{j-1}, x_{j}, x_{j+1}, \ldots, x_{n}\right)=\varphi\left(x_{0}, \ldots, x_{j-1}, x_{j+1}, \ldots, x_{n}\right), \quad 0 \leq j \leq n, \\
& \left(\eta^{j}(\varphi)\right)\left(x_{0}, \ldots, x_{n-1}\right)=\varphi\left(x_{0}, \ldots, x_{j-1}, x_{j}, x_{j}, \ldots, x_{n-1}\right), \quad 0 \leq j \leq n-1 .
\end{aligned}
$$

Likewise, on the degree $n$ constituent $\operatorname{Map}\left(G^{\times(n+1)}, V\right)$, the right $R G$-module structure is given by the association

$$
\begin{aligned}
& \operatorname{Map}\left(G^{\times(n+1)}, V\right) \times G \longrightarrow \operatorname{Map}\left(G^{\times(n+1)}, V\right),(\alpha, x) \mapsto \alpha \cdot x, \\
& (\alpha \cdot x)\left(x_{0}, x_{1}, \ldots, x_{n}\right)=\left(\alpha\left(x x_{0}, x x_{1}, \ldots, x x_{n}\right)\right) x,
\end{aligned}
$$

where $\alpha \in \operatorname{Map}\left(G^{\times(n+1)}, \square V\right)$ and $x, x_{0}, \ldots, x_{n} \in G$. Together with the injection $\omega_{V}$, the resulting chain complex $|\mathbf{U}(V)|$ is an injective resolution of $V$ in the category of right $R G$-modules.

PROPOSITION 2.8. The natural isomorphism of monads mentioned in Corollary 2.6 induces a natural isomorphism

$$
\Theta: \mathbf{U}(V)=\left(\operatorname{Map}\left(G^{\times(n+1)}, V\right), \varepsilon^{j}, \eta^{j}\right)_{n \in \mathbb{N}} \longrightarrow\left(\operatorname{Map}\left(G^{\times(n+1)}, \square V\right), \varepsilon^{j}, \eta^{j}\right)_{n \in \mathbb{N}}=\mathbf{T}(V)
$$

of cosimplicial objects in the category of right $R G$-modules and hence a natural isomorphism between the resulting injective resolutions of $V$ in the category of right $R G$-modules. In degree $n \geq 0$, this isomorphism is given by the association

$$
\begin{aligned}
& \vartheta_{n}: \operatorname{Map}\left(G^{\times(n+1)}, V\right) \longrightarrow \operatorname{Map}\left(G^{\times(n+1)}, \square V\right) \\
& \vartheta_{n}(\alpha)\left(x_{0}, x_{1}, \ldots, x_{n}\right)=\alpha\left(x_{0}, x_{0} x_{1}, \ldots, x_{0} x_{1} \ldots x_{n-1}, x_{0} x_{1} \ldots x_{n}\right) \cdot x_{0} \ldots x_{n}
\end{aligned}
$$

where $x_{0}, x_{1}, \ldots x_{n} \in G$ and $\alpha \in \operatorname{Map}\left(G^{\times(n+1)}, V\right)$.

Proof. Only the statement making the isomorphism explicit requires proof. For $n=0$, the given formula reduces to the commutativity of diagram (2.8) above. For $n \geq 1$, the verification is straightforward and left to the reader. A conceptual explanation will be given in Section 4 below. 
EXAMPLE 2.9. This example is the variant of Example 2.3 for Lie groups rather than just discrete groups and smooth maps rather than just set maps; it is related to Example 2.2 in the same way as Example 2.3 is related to Example 2.1. Let the ground ring be that of the reals, $\mathbb{R}$, and let $G$ be a Lie group. Further, let $\mathcal{U}: \operatorname{Mod}_{G} \longrightarrow \operatorname{Mod}_{G}$ be the functor which assigns to the right $G$-representation $V$ the right $G$-representation $\mathcal{U}(V)=C^{\infty}(G, V)$, endowed with the right diagonal action

$$
C^{\infty}(G, V) \times G \longrightarrow C^{\infty}(G, V), \quad(\rho, x) \longmapsto \rho \cdot x
$$

given by

$$
(\rho \cdot x)(y)=(\rho(x y)) x, \quad x, y \in G, \quad \rho: G \rightarrow V,
$$

let $\omega$ be the natural transformation given by the assignment to the right $G$-representation $V$ of

$$
\omega=\omega_{V}: V \longrightarrow C^{\infty}(G, V), \quad v \longmapsto \omega_{v}: G \rightarrow V, \quad \omega_{v}(x)=v, \quad v \in V, \quad x \in G,
$$

and let

$$
v: \mathcal{U}^{2} \longrightarrow \mathcal{U}
$$

be the natural transformation given by the assignment to the right $G$-representation $V$ of the association

$$
v_{V}: C^{\infty}\left(G, C^{\infty}(G, V)\right) \longrightarrow C^{\infty}(G, V), \quad\left(\nu_{V}(\Psi)\right)(x)=\Psi(x)(x), \quad x \in G,
$$

where $\Psi$ ranges over smooth maps of the kind $G \rightarrow C^{\infty}(G, V)$. The system $(\mathcal{U}, \omega, v)$ is a monad over the category $\operatorname{Mod}_{G}$; it is, indeed, an alternate description of the monad $(\mathcal{T}, u, \mu)$ in Example 2.2. More precisely, as in the situation of Example 2.3, the natural transformation $\vartheta$ which, to the right $G$-representation $V$, assigns the isomorphism

$$
\vartheta_{V}: C^{\infty}(G, V) \longrightarrow C^{\infty}(G, \square V)
$$

of right $G$-modules given by

$$
(\vartheta(\rho))(y)=(\rho(y)) y
$$

yields an isomorphism of monads from the monad $(\mathcal{U}, \omega, v)$ to the monad $(\mathcal{T}, u, \mu)$ in Example 2.2.

Given the differentiable right $G$-module $V$, the dual standard construction

$$
\mathbf{U}(V)=\left(C^{\infty}\left(G^{\times(n+1)}, V\right), \varepsilon^{j}, \eta^{j}\right)_{n \in \mathbb{N}}
$$


associated with $V$ and the monad $(\mathcal{U}, \omega, v)$ is a cosimplicial object in the category of differentiable right $G$-modules, and the chain complex $|\mathbf{U}(V)|$ associated with this cosimplicial object, together with the injection $\omega_{V}$, is a differentiably injective resolution of $V$ in the category of differentiable right $G$-modules. Apart from the fact that we deal with differentiable right $G$-modules rather than differentiable left $G$-modules, the resolution $|\mathbf{U}(V)|$ is, in fact, precisely the homogeneous resolution of $V$, cf. p. 371 of [19].

The association (2.12), now interpreted in the smooth category, that is, with $C^{\infty}$ substituted for Map, yields the isomorphism

$$
|\Theta|:|\mathbf{U}(V)| \longrightarrow|\mathbf{T}(V)|
$$

of differentiably injective resolutions of $V$ in the category of differentiable right $G$-modules, where $\mathbf{T}(V)$ is the cosimplicial object (2.5).

Let $\mathfrak{g}$ be a Lie algebra over a general ground ring $R$. Recall that, as a graded Lie algebra, the cone $C \mathfrak{g}$ in the category of differential graded $R$-Lie algebras is defined to be the semi-direct product $s \mathfrak{g} \rtimes \mathfrak{g}$ of $\mathfrak{g}$ with the suspension $s \mathfrak{g}$; here the suspension $s \mathfrak{g}$ is just $\mathfrak{g}$ itself, but regraded up by 1 , and $s \mathfrak{g}$ is considered as an abelian graded Lie algebra concentrated in degree 1. The differential $d$ is given by $d(s Y)=Y$, where $Y \in \mathfrak{g}$. The cone $C \mathfrak{g}$ is a contractible differential graded $R$-Lie algebra. See [29] for details.

EXAMPLE 2.10. Let $R$ be an arbitrary commutative ring with 1 and $\mathfrak{g}$ an $R$-Lie algebra, which we suppose to be projective as an $R$-module. Let $C \mathfrak{g}$ be the cone on $\mathfrak{g}$ in the category of differential graded Lie algebras. Let $\mathcal{D}=\mathcal{C}_{\mathfrak{g}}$, the category of right $\mathfrak{g}$-chain complexes, let $\mathcal{M}=\operatorname{Mod}_{C \mathfrak{g}}$, and let $\mathcal{G}_{C \mathfrak{g}}^{\mathfrak{g}}: \mathcal{C}_{\mathfrak{g}} \rightarrow \operatorname{Mod}_{C \mathfrak{g}}$ be the functor given by

$$
\mathcal{G}_{C \mathfrak{g}}^{\mathfrak{g}}(V)=\operatorname{Hom}_{\mathfrak{g}}(\mathrm{U}[C \mathfrak{g}], V) \cong(\operatorname{Alt}(\mathfrak{g}, V), d),
$$

the total object arising from the bicomplex having $\mathrm{Alt}^{*}\left(\mathfrak{g}, V_{*}\right)$ as underlying bigraded $R$-module; here $V$ ranges over right $\mathfrak{g}$-chain complexes, $(\operatorname{Alt}(\mathfrak{g}, V), d)$ is endowed with the obvious right $(C \mathfrak{g})$-module structure coming from the obvious left $(\mathrm{U}[C \mathfrak{g}])$-module structure on itself or, equivalently, that given by the operations of contraction and Lie derivative on the CCE complex $(\operatorname{Alt}(\mathfrak{g}, V), d)$, cf. [29]. The functor $\mathcal{G}_{C \mathfrak{g}}^{\mathfrak{g}}$ is right adjoint to the forgetful functor $\square: \operatorname{Mod}_{C \mathfrak{g}} \rightarrow \mathcal{C}_{\mathfrak{g}}$ and hence defines a monad $(\mathcal{T}, u, \mu)$ over the category $\operatorname{Mod}_{C \mathfrak{g}}$. Given the right $(C \mathfrak{g})$ module $\mathbf{V}$, the chain complex $|\mathbf{T}(\mathbf{V})|$ arising from the dual standard construction $\mathbf{T}(\mathbf{V})$ associated with $\mathbf{V}$ is a resolution of $\mathbf{V}$ in the category of $(C \mathfrak{g})$-modules that is injective relative to the category $\mathcal{C}_{\mathfrak{g}}$ of right $\mathfrak{g}$-chain complexes. Given a right $(C \mathfrak{g})$ module $\mathbf{W}$, the relative differential $\operatorname{Ext}_{(C \mathfrak{g}, \mathfrak{g})}(\mathbf{W}, \mathbf{V})$ is the homology of the chain complex 
In particular, for $\mathbf{W}=R$, the relative differential graded $\operatorname{Ext}_{(C \mathfrak{g}, \mathfrak{g})}(R, \mathbf{V})$ is the homology of the chain complex $|\mathbf{T}(\mathbf{V})|^{C \mathfrak{g}}$.

EXAMPLE 2.11. This example extends Example 2.9. Let the ground ring again be that of the reals, $\mathbb{R}$, and let $G$ be a Lie group. We will now switch to differentiable left $G$-modules. Let $\xi: P \rightarrow B$ be a right principal $G$-bundle. Let $\mathcal{U}_{\xi}:{ }_{G} \operatorname{Mod} \longrightarrow$ ${ }_{G}$ Mod be the functor which assigns to the left $G$-representation $V$ the left $G$-representation $\mathcal{U}_{\xi}(V)=C^{\infty}(P, V)$, endowed with the left diagonal action

$$
G \times C^{\infty}(P, V) \longrightarrow C^{\infty}(P, V), \quad(x, \rho) \longmapsto x \cdot \rho
$$

given by

$$
(x \cdot \rho)(y)=x(\rho(y x)), \quad x \in G, \quad y \in P, \quad \rho: P \rightarrow V,
$$

let $\omega$ be the natural transformation given by the assignment to the left $G$-representation $V$ of

$$
\omega=\omega_{V}: V \longrightarrow C^{\infty}(P, V), \quad v \longmapsto \omega_{v}: P \rightarrow V, \quad \omega_{v}(y)=v, \quad v \in V, \quad y \in P,
$$

and let

$$
v: \mathcal{U}_{\xi}^{2} \longrightarrow \mathcal{U}_{\xi}
$$

be the natural transformation given by the assignment to the left $G$-representation $V$ of the association

$$
v_{V}: C^{\infty}\left(P, C^{\infty}(P, V)\right) \longrightarrow C^{\infty}(P, V), \quad\left(v_{V}(\Psi)\right)(y)=\Psi(y)(y), \quad y \in P,
$$

where $\Psi$ ranges over smooth maps of the kind $P \rightarrow C^{\infty}(P, V)$. The system $\left(\mathcal{U}_{\xi}, \omega, v\right)$ is a monad over the category ${ }_{G}$ Mod.

When $P$ is just $G$, so that $\xi$ is the trivial principal $G$-bundle over a point, the monad $\left(\mathcal{U}_{\xi}, \omega, v\right)$ plainly reduces to the monad $(\mathcal{U}, \omega, v)$ spelled out in Example 2.3, phrased in the category of differentiable left $G$-modules rather than differentiable right $G$-modules.

Given the differentiable left $G$-module $V$, the dual standard construction

$$
\mathbf{U}_{\xi}(V)=\left(C^{\infty}\left(P^{\times(n+1)}, V\right), \varepsilon^{j}, \eta^{j}\right)_{n \in \mathbb{N}}
$$

associated with $V$ and the monad $\left(\mathcal{U}_{\xi}, \omega, v\right)$ is a cosimplicial object in the category of differentiable left $G$-modules, and the chain complex $\left|\mathbf{U}_{\xi}(V)\right|$ associated with this cosimplicial object, together with the injection $\omega_{V}$, is a differentiably injective resolution of $V$ in the category of differentiable left $G$-modules. In particular, the obvious restriction map from $\left|\mathbf{U}_{\xi}(V)\right|$ to the chain complex $|\mathbf{U}(V)|$ associated with the cosimplicial object (2.18) but phrased for differentiable left $G$-modules rather 
than differentiable right ones, is a comparison map between the two resolutions. For the special case where $\xi$ is the trivial principal bundle, the obvious map from $|\mathbf{U}(V)|$ to $\left|\mathbf{U}_{\xi}(V)\right|$ is a comparison map in the other direction. In the general case, using (i) an open cover of $B$ such that $\xi$ is trivial on each member of the cover and, furthermore, (ii) a smooth partition of unity subordinate to this cover, we can still construct a comparison map from $|\mathbf{U}(V)|$ to $\left|\mathbf{U}_{\xi}(V)\right|$.

For intelligibility we recall that, given a discrete group $\pi$ and a $\pi$-module $V$, for any free $\pi$-set $\Gamma$, the injection

$$
V \longrightarrow \operatorname{Map}(\Gamma, V), \quad v \longmapsto \varphi_{v}, \quad \varphi_{v}(y)=v, \quad v \in V, \quad y \in \Gamma,
$$

is an injection of $V$ into a relatively injective $\pi$-module, $\operatorname{Map}(\Gamma, V)$ being suitably turned into a $\pi$-module. For our purposes, $C^{\infty}(P, V)$ has formally the same significance as a relatively injective $\pi$-module of the kind $\operatorname{Map}(\Gamma, V)$.

\section{Differentiable Cohomology over a Lie Groupoid}

We shall see that the monads spelled out in Examples 2.3 and 2.9 (and written as $(\mathcal{U}, \omega, \nu))$ generalize to groupoids and yield the appropriate injective resolutions while the monads described in Examples 2.1 and 2.2 do not extend in an obvious manner to groupoids. See also Remark 3.1 below.

Our reference for terminology is [8], [30]-[31]; we will use the conventions in [8]. Let $\Omega$ be a Lie groupoid; we denote the smooth manifold of objects by $B_{\Omega}$, the source and target maps by $s: \Omega \rightarrow B_{\Omega}$ and $t: \Omega \rightarrow B_{\Omega}$, respectively, and the object inclusion map by 1: $B_{\Omega} \rightarrow \Omega$. Thus we view the element $u$ of $\Omega$ as an arrow from $s(u)$ to $t(u)$. According to the conventions in [8] and [32], the elements $u, v$ of $\Omega$ are composable when $s(u)=t(v) \in B_{\Omega}$, and we then write the element of $\Omega$ arising from composition simply as $u v$. Often we do not distinguish in notation between the groupoid and its space of (iso)morphisms. Given $p, q \in B_{\Omega}$ we will denote the $t$-fiber $t^{-1}(p)$ by $\Omega_{p}$ (beware: in [32] the $t$-fiber is written as $\Omega^{p}$ ), the $s$-fiber $s^{-1}(q)$ by $\Omega^{q}$ (beware: in [32] the $s$-fiber is written as $\Omega_{q}$ ) and we write

$$
\Omega_{p}^{q}=\Omega_{p} \cap \Omega^{q}
$$

thus $\Omega_{q}^{q}$ is the vertex group at $q$. Moreover, given an object $q$, the groupoid composition amounts to a smooth map

$$
\Omega^{q} \times \Omega_{q} \longrightarrow \Omega, \quad(u, v) \mapsto u v .
$$

We suppose throughout that $\Omega$ is locally trivial in the sense that the smooth map

$$
(t, s): \Omega \longrightarrow B_{\Omega} \times B_{\Omega}
$$

is a submersion. Then $\Omega$ is locally trivial in the usual sense; see p. 16 of [31] for details. Furthermore, for any $p \in B_{\Omega}$, the projection $s: \Omega_{p} \rightarrow B_{\Omega}$ is then a principal 
left $\Omega_{p}^{p}$-bundle and, for any $q \in B_{\Omega}$, the projection $t: \Omega^{q} \rightarrow B_{\Omega}$ is a principal right $\Omega_{q}^{q}$-bundle. For any object $q$, the smooth map (3.1) induces a diffeomorphism from $\Omega^{q} \times \Omega_{q}^{q} \Omega_{q}$ onto $\Omega$, and the target map $t: \Omega \rightarrow B_{\Omega}$ thus arises as the fiber bundle

$$
t: \Omega^{q} \times \Omega_{q}^{q} \Omega_{q} \longrightarrow B_{\Omega}
$$

associated with the principal right $\Omega_{q}^{q}$-bundle $t: \Omega^{q} \rightarrow B_{\Omega}$ and the left $\Omega_{q}^{q}$-action on $\Omega_{q}$; likewise the source map $s: \Omega \rightarrow B_{\Omega}$ arises as the fiber bundle

$$
s: \Omega^{q} \times \Omega_{q}^{q} \Omega_{q} \longrightarrow B_{\Omega}
$$

associated with the principal left $\Omega_{q}^{q}$-bundle $s: \Omega_{q} \rightarrow B_{\Omega}$ and the right $\Omega_{q}^{q}$-action on $\Omega^{q}$.

It is well known, cf. [31] (Theorem 1.6.5), that any locally trivial Lie groupoid is the gauge groupoid of an associated principal bundle: Let $G$ be a Lie group and $\xi: P \rightarrow B$ a principal right $G$-bundle. The gauge groupoid $((P \times P) / G, B, s, t, 1)$ of $\xi$ arises from the product groupoid or pair groupoid $(P \times P, P, s, t, 1)$ having composition given by the association

$$
(u, v)(v, w)=(u, w), \quad u, v, w \in P
$$

in the obvious way as indicated. Given the locally trivial Lie groupoid $\Omega$, pick $q \in$ $B_{\Omega}$, let $G=\Omega_{q}^{q}$, and let

$$
\xi=t: \Omega^{q} \longrightarrow B_{\Omega} ;
$$

as noted above, $\xi$ is a principal right $G$-bundle; the groupoid composition (3.1) can then be written as

$$
\Omega^{q} \times \Omega^{q} \longrightarrow \Omega, \quad(u, v) \mapsto u v^{-1},
$$

and this association induces an isomorphism between the gauge groupoid of $\xi$ and $\Omega$.

Given two spaces $f_{1}: X_{1} \rightarrow Y$ and $f_{2}: X_{2} \rightarrow Y$ over the space $Y$, we will use the notation

$$
X_{1} \times f_{1}, Y, f_{2} X_{2}=\left\{\left(x_{1}, x_{2}\right) ; f_{1}\left(x_{1}\right)=f_{2}\left(x_{2}\right) \in Y\right\} \subseteq X_{1} \times X_{2} .
$$

Thus $X_{1} \times f_{1}, Y, f_{2} X_{2}$ is the fiber product of $X_{1}$ and $X_{2}$ over $Y$.

Let $M$ be a smooth manifold and let $f: M \rightarrow B_{\Omega}$ be a smooth manifold over $B_{\Omega}$, that is, $f$ is a smooth map. A smooth left action of $\Omega$ on $f$, cf. [8] (p. 101), is given by a commutative diagram

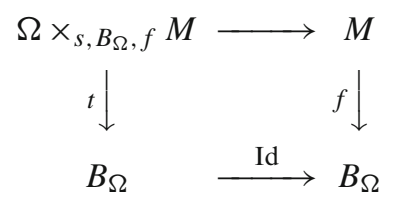


such that the obvious associativity constraint is satisfied. We will then say that $f$ is a smooth left $\Omega$-manifold. The notions of smooth right action

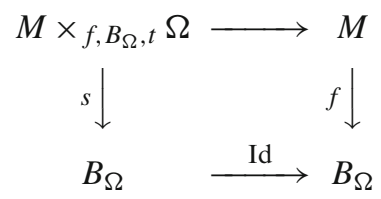

of $\Omega$ on $f$ and of smooth right $\Omega$-manifold are defined accordingly. Morphisms of left $\Omega$-manifolds and morphisms of right $\Omega$-manifolds are defined in the obvious way, and left $\Omega$-manifolds as well as right $\Omega$-manifolds constitute a category.

An ordinary group acts on itself by left translation and by right translation. Accordingly, left translation in $\Omega$ induces a left action of $\Omega$ on $f=t: \Omega \rightarrow B_{\Omega}$, viewed here as a fiber bundle having, over $q \in B_{\Omega}$, fiber $\Omega_{q}$. Indeed, in view of the previous discussion, after a choice of $q \in B_{\Omega}$ has been made, $t: \Omega \rightarrow B_{\Omega}$ acquires the structure of a fiber bundle associated with the right principal $\Omega_{q}^{q}$-bundle $t: \Omega^{q} \rightarrow B_{\Omega}$ and the left translation action of $\Omega_{q}^{q}$ on $\Omega_{q}$. In the same vein, right translation in $\Omega$ induces a right action of $\Omega$ on $f=s: \Omega \rightarrow B_{\Omega}$ viewed as a fiber bundle having, over $p \in B_{\Omega}$, fiber $\Omega^{p}$; after a choice of $p \in B_{\Omega}$ has been made, we can view $s$ as a fiber bundle associated with the left principal $\Omega_{p}^{p}$-bundle $s: \Omega_{p} \rightarrow B_{\Omega}$ via the right translation action of $\Omega_{p}^{p}$ on $\Omega_{p}$.

In particular, let $\zeta: E \rightarrow B_{\Omega}$ be a vector bundle. A representation of $\Omega$ on $\zeta$ from the left or, synonymously, a left linear action of $\Omega$ on $\zeta$, is defined in the obvious manner as a left action of $\Omega$ on $\zeta$ that is linear in the obvious sense, and we then refer to $\zeta$ as a differentiable left $\Omega$-module. Morphisms of left $\Omega$-modules are defined in the obvious way, and left $\Omega$-modules constitute a category which we will denote by $\Omega$ Mod. We do not consider right $\Omega$-modules.

Let $\zeta: E \rightarrow B_{\Omega}$ be a vector bundle endowed with a left $\Omega$-module structure. The construction in (2.3) of [32] yields a differentiably injective left $\Omega$-module

$$
F(\Omega, \zeta): F(\Omega, E) \longrightarrow B_{\Omega},
$$

that is, $F(\Omega, \zeta)$ is the projection map of a vector bundle over $B_{\Omega}$ together with (i) a left $\Omega$-module structure

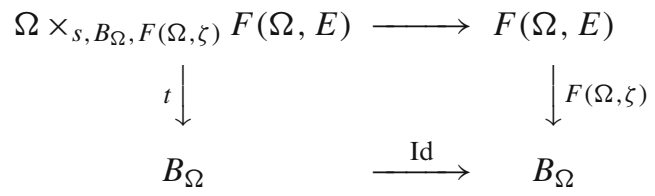

of $\Omega$ on $F(\Omega, \zeta)$ and (ii) a canonical injection $\omega_{\zeta}: E \rightarrow F(\Omega, \zeta)$ such that the diagram

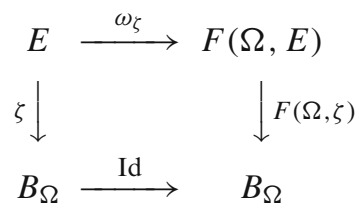


is a morphism of left $\Omega$-modules; furthermore, $F(\Omega, \cdot)$ and $\omega$ are natural in the $\Omega$-module variable, that is, $F(\Omega, \cdot)$ is an endofunctor of ${ }_{\Omega} \operatorname{Mod}$ and $\omega$ is a natural transformation $\mathcal{I} \rightarrow F(\Omega, \cdot)$. More precisely, the construction has the following structural properties:

1. For $q \in B_{\Omega}$, the fiber $(F(\Omega, \zeta))^{-1}(q) \subseteq F(\Omega, E)$ is the space $C^{\infty}\left(\Omega^{q}, E_{q}\right)$;

2. the embedding $\omega_{\zeta}$ is the constant one in the sense that, for $q \in B_{\Omega}$, the restriction

$$
\left.\omega_{\zeta}\right|_{E_{q}}: E_{q} \longrightarrow C^{\infty}\left(\Omega^{q}, E_{q}\right)
$$

sends $v \in E_{q}$ to the constant map which assigns $v \in E_{q}$ to $x \in \Omega^{q}$;

3. the left $\Omega$-action on $F(\Omega, \zeta)$ is the action arising from right translation in $\Omega$ and from the left $\Omega$-action on $\zeta$.

The left $\Omega$-action on $F(\Omega, \zeta)$ with respect to right translation in $\Omega$ and with respect to the left $\Omega$-action on $\zeta$ is given by that action which, in the group case, corresponds to the diagonal action given by a formula of the kind (2.20). Appropriately adjusted to the present situation, this formula leads to the following description of the action:

$$
(x \cdot \rho)(u)=x(\rho(u x)), \quad x \in \Omega_{q}, \quad u \in \Omega^{q}, \quad \rho: \Omega^{s(x)} \rightarrow E_{s(x)}, \quad q \in B_{\Omega} .
$$

Indeed, the situation can be depicted by means of the commutative diagram

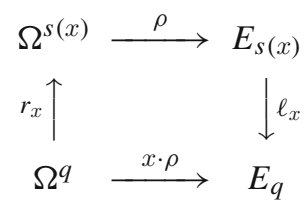

where $\ell_{x}$ refers to left translation in $E$ with $x$ and $r_{x}$ to right translation in $\Omega$ with $x$. The formula (3.8) is exactly the same as (3.5) in [32].

Remark 3.1. The diagram (3.9) explains in particular why the $\Omega$-action on $F(\Omega, \zeta)$ cannot be defined by right translation in $\Omega$ alone, that is to say, why the action in Example 2.1 and in Example 2.3 does not extend to groupoids since the putative extension of this kind of action would not be compatible with the variance constraint imposed by the groupoid axioms. See also the Remark on p. 285 of [32].

Remark 3.2. The construction of $F(\Omega, \zeta)$ has been carried out in [32] for locally trivial locally compact topological groupoids rather than Lie groupoids. The construction given in [32] carries over to Lie groupoids as well. We leave the details to the reader.

The previous discussion entails the following observation which unravels the structure of $F(\Omega, \zeta)$ : 
PROPOSITION 3.3. Given the left $\Omega$-module $\zeta: E \rightarrow B_{\Omega}$, for any $q \in B_{\Omega}$, the left $\Omega$-module structure on $F(\Omega, \zeta)$ induces a diffeomorphism

$$
\Omega^{q} \times{ }_{\Omega_{q}^{q}} C^{\infty}\left(\Omega^{q}, E_{q}\right) \longrightarrow F(\Omega, E)
$$

over $B_{\Omega}$. Thus, suppose that $G$ is a Lie group, that $\xi: P \rightarrow B_{\Omega}$ is a principal right $G$-bundle having $\Omega$ as its gauge groupoid, and that, as a vector bundle, $\zeta$ is the vector bundle associated with $\xi$ and the left G-representation $V$. Then, as a vector bundle, $F(\Omega, \zeta)$ may be taken to be the vector bundle

$$
\xi \times{ }_{G} C^{\infty}(P, V): P \times{ }_{G} C^{\infty}(P, V) \longrightarrow B_{\Omega}
$$

associated with $\xi$ and the diagonal left $G$-module structure (2.20) on $C^{\infty}(P, V)$ coming from the right $G$-action on $P$ and the left $G$-action on $V$, and the left $\Omega$-module structure on $F(\Omega, \zeta)$ is the familiar left $\Omega$-module structure on the associated vector bundle (3.11).

The "familiar left $\Omega$-structure on an associated fiber bundle" is explained, e.g., in Theorem 1.6 .5 (p. 35) of [31].

We will now write the endofunctor $F(\Omega, \cdot)$ on $\operatorname{Mod}_{\Omega}$ as $\mathcal{U}: \operatorname{Mod}_{\Omega} \longrightarrow \operatorname{Mod}_{\Omega}$. Let

$$
v: \mathcal{U}^{2} \longrightarrow \mathcal{U}
$$

be the obvious extension of the natural transformation (2.16). Thus $v$ is the natural transformation given by the assignment to the left $\Omega$-module $\zeta$ of the morphism

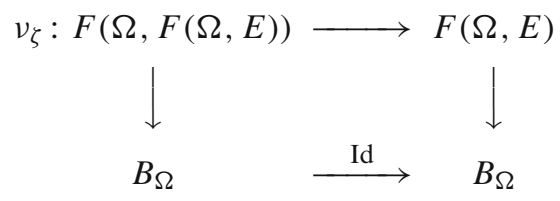

of vector bundles which, over $q \in B_{\Omega}$, sends $\Psi: \Omega^{q} \rightarrow C^{\infty}\left(\Omega^{q}, E_{q}\right)$ to $v_{q}(\Psi)$ given by $v_{q}(\Psi)(x)=\Psi(x)(x)$, where $x \in \Omega^{q}$.

Remark 3.4. The definition of the natural transformation $v$ fails to work with a putative construction involving all the (smooth) maps from $\Omega$ to $E$ over $B_{\Omega}, \Omega$ being viewed over $B_{\Omega}$ through $t: \Omega \rightarrow B_{\Omega}$, rather than $F(\Omega, E)$ since, as pointed out above, the diagonal map for groups does not extend to a diagonal map for general groupoids. Thus, to define cohomology via an appropriate cosimplicial object, we cannot simply take functions on an associated simplicial object of the kind $(E \Omega)^{\text {left }}$ explored in the next section unless the groupoid under discussion is a group, cf. Remark 4.6. 
The system $(\mathcal{U}, \omega, v)$ is a monad over the category $\Omega$ Mod. Given the left $\Omega$-module $\zeta$, the dual standard construction $\mathbf{U}(\zeta)$ associated with $(\mathcal{U}, \omega, v)$ and the left $\Omega$-module $\zeta$ is a cosimplicial object in the category of left $\Omega$-modules; the chain complex $|\mathbf{U}(\zeta)|$ associated with $\mathbf{U}(\zeta)$, together with the injection $\omega_{\zeta}$, is then a differentiably injective resolution of $\zeta$ in the category of left $\Omega$-modules. The resulting non-normalized chain complex coincides with the standard resolution (5.4) in [32], developed there for locally trivial locally compact topological groupoids. In view of our categorical point of view that a monad defines a relative Ext, that observation establishes the following.

THEOREM 3.5. Let $\mathcal{C}$ be the category of continuous vector bundles on $B_{\Omega}$ with split morphisms. Given two left $\Omega$-modules $\eta$ and $\zeta$, the chain complex

$\operatorname{Hom}_{\Omega}(\eta,|\mathbf{U}(\zeta)|)$

defines the relative $\operatorname{Ext}_{(\Omega, \mathcal{C})}(\eta, \zeta)$; in particular, when we take $\eta$ to be the trivial real line bundle on $B_{\Omega}$ with trivial left $\Omega$-module structure, this Ext reduces to the cohomology of $\Omega$ with values in $\zeta$ introduced in [32].

In the special case where $\Omega$ is an ordinary Lie group, say $G$, and when $\eta$ is the trivial $G$-representation $\mathbb{R}$, the functor $\operatorname{Ext}_{(\Omega, \mathcal{C})}(\mathbb{R}, \cdot)$ reduces to the cohomology theory developed in [19] and reproduced in Section 2 above.

We will now unravel the functor $|\mathbf{U}(\zeta)|$ in terms of a corresponding principal bundle: Let $\xi: P \rightarrow B_{\Omega}$ be a principal right $G$-bundle, such that the associated gauge groupoid is isomorphic to $\Omega$. We can pick any $q \in B_{\Omega}$ and take $G$ to be the vertex group $\Omega_{q}^{q}$; then the projection $t: \Omega^{q} \rightarrow B_{\Omega}$ is such a principal right $G$-bundle.

Let $\zeta: E \rightarrow B_{\Omega}$ be a left $\Omega$-module. Relative to $\xi$, as a topological vector bundle, $\zeta$ amounts to an induced vector bundle of the kind

$$
P \times{ }_{G} V \longrightarrow B_{\Omega}
$$

where $V$ is a topological vector space endowed with a left $G$-representation, so that the standard formalism of associated fiber bundles applies. Then, relative to $\xi$, as a topological vector bundle, $F(\Omega, \zeta)$ amounts to an induced vector bundle of the kind

$$
F(\Omega, \zeta): P \times{ }_{G} C^{\infty}(P, V) \longrightarrow B_{\Omega},
$$

the vector space $C^{\infty}(P, V)$ being suitably topologized and made into a left $G$-representation via the left action (2.20), the requisite left $\Omega$-module structure on the induced vector bundle $F(\Omega, \zeta)$ being that induced from the left $G$-structure (2.20).

Let $\left|\mathbf{U}_{\xi}(V)\right|$ be the injective resolution of the differentiable left $G$-module arising from the cosimplicial object (2.24). Now, relative to $\xi$, as a topological differential graded vector bundle, $|\mathbf{U}(\zeta)|$ amounts to an induced differential graded vector 
bundle of the kind

$$
P \times_{G}\left|\mathbf{U}_{\xi}(V)\right| \longrightarrow B_{\Omega},
$$

the differential graded left $G$-module $\left|\mathbf{U}_{\xi}(V)\right|$ being suitably topologized.

The following result, established originally as Theorem 3 of [32] for the rigid cohomology of a general locally trivial topological groupoid, is now immediate for the present cohomology theory of locally trivial Lie groupoids; we spell it out for later reference, since we will establish a formally similar result, but for equivariant cohomology rather than just differentiable cohomology:

PROPOSITION 3.6. Restriction induces an isomorphism of the cohomology of $\Omega$ with values in $\zeta$ onto the Hochschild-Mostow differentiable cohomology $\mathrm{H}(G, V)$ of $G$ with values in $V$.

Proof. Let $V_{1}$ and $V_{2}$ be two left $G$-representations and let $\vartheta_{1}: P \times_{G} V_{1} \rightarrow B_{\Omega}$ and $\vartheta_{2}: P \times_{G} V_{2} \rightarrow B_{\Omega}$ be the corresponding left $\Omega$-modules. In view of an observation in [38], $\operatorname{Hom}_{\Omega}\left(\zeta_{1}, \zeta_{2}\right)$ is naturally isomorphic to $\operatorname{Hom}_{G}\left(V_{1}, V_{2}\right)$. More precisely, the assignment to a left $G$-representation $V$ of the $\Omega$-module $\vartheta: P \times_{G} V \rightarrow B_{\Omega}$ extends to a functor which is left-adjoint to the restriction functor $\Omega_{\Omega} \operatorname{Mod} \rightarrow{ }_{G} \operatorname{Mod}$. Hence, when $\eta$ refers to the trivial line bundle on $B_{\Omega}$ with trivial $\Omega$-module structure, the chain complex $\operatorname{Hom}_{\Omega}\left(\eta,\left|\mathbf{U}_{\xi}(\zeta)\right|\right)$ reduces to the chain complex $\operatorname{Hom}_{G}\left(\mathbb{R},\left|\mathbf{U}_{\xi}(V)\right|\right) \cong\left|\mathbf{U}_{\xi}(V)\right|^{G}$ calculating $\mathrm{H}(G, V)$.

\section{Comonads and Standard Constructions}

Recall that any object $Y$ of a symmetric monoidal category endowed with a cocommutative diagonal - we will take the categories of spaces, of smooth manifolds, of groups, of vector spaces, of Lie algebras, etc., - defines two simplicial objects in the category, the trivial object which, by an abuse of notation, we still write as $Y$, and the total object EY ("total object" not being standard terminology in this generality); the trivial object $Y$ has a copy of $Y$ in each degree and all simplicial operations are the identity while, for $p \geq 0$, the degree $p$ constituent $E Y_{p}$ of the total object $E Y$ is a product of $p+1$ copies of $Y$ with the familiar face operations given by omission and degeneracy operations given by insertion. See e.g., [5] and [28] (1.1). When $Y$ is an ordinary $R$-module, the simplicial $R$-module associated with $Y$ is in fact the result of application of the Dold-Kan functor $D K$ from chain complexes to simplicial $R$-modules, cf. e.g., [10] (3.2 on p. 219).

For illustration, let $\mathfrak{g}$ be a Lie algebra which we suppose to be projective as a module over the ground ring $R$. The total object $E \mathfrak{g}$ associated with $\mathfrak{g}$ in the category of Lie algebras relative to the obvious monoidal structure is a simplicial Lie algebra. This construction plays a major role in the predecessor [29] of the present paper and is lurking behind some of the constructions in the next section. 
Likewise let $G$ be a group. When the group $G$ is substituted for $Y$, the resulting simplicial object is a simplicial group $E G$, and the diagonal injection $G \rightarrow E G$ turns $E G$ into a simplicial principal right (or left) $G$-set. We will refer to $E G$ as the homogeneous universal object for $G$.

Let $V$ be a right $R G$-module. The simplicial structure of $E G$ and the degreewise right diagonal $R G$-module structures (2.6) (with $E G$ substituted for $G$ ), relative to the right translation $G$-action on $E G$ where $G$ is viewed as a subgroup of $E G$ and relative to the $R G$-module structure on $V$, turn

$$
\operatorname{Map}(E G, V),
$$

into a cosimplicial object in the category of right $R G$-modules. The proof of the following proposition is straightforward.

PROPOSITION 4.1. As a cosimplicial object in the category of right RG-modules, $\operatorname{Map}(E G, V)$ coincides with $\mathbf{U}(V)$ (introduced as (2.10) above).

Let $(E G)^{\text {left }}$ be the simplicial group having the iterated semi-direct product

$$
(E G)_{n}^{\text {left }}=G \ltimes G \ltimes \cdots \ltimes G(n+1 \text { copies of } G)
$$

as degree $n$ constituent; the nonhomogeneous face operators $\partial_{j}$ are given by the familiar formulas

$$
\begin{aligned}
& \partial_{j}\left(x_{0}, x_{1}, \ldots, x_{n}\right)=\left(x_{0}, \ldots, x_{j-2}, x_{j-1} x_{j}, x_{j+1}, \ldots, x_{n}\right) \quad(0 \leq j<n) \\
& \partial_{n}\left(x_{0}, x_{1}, \ldots, x_{n}\right)=\left(x_{0}, \ldots, x_{n-1}\right)
\end{aligned}
$$

and the nonhomogeneous degeneracy operators $s_{j}$ are given by

$$
s_{j}\left(x_{0}, x_{1}, \ldots, x_{n}\right)=\left(x_{0}, \ldots, x_{j-1}, e, x_{j}, \ldots, x_{n}\right) \quad(0 \leq j \leq n) .
$$

The associations

$$
\left(x_{0}, x_{1}, \ldots, x_{n}\right) \longmapsto\left(x_{0}, x_{0} x_{1}, \ldots, x_{0} x_{1} x_{2} \ldots x_{n-1}, x_{0} x_{1} x_{2} \ldots x_{n}\right), x_{j} \in G,
$$

as $n$ ranges over the natural numbers, induce an isomorphism of simplicial groups

$$
(E G)^{\text {left }} \longrightarrow E G \text {. }
$$

We will refer to $(E G)^{\text {left }}$ as the nonhomogeneous left universal object for $G$. Associations of the kind (4.4) (for the case of a discrete group) can be found, e.g., in [34] (Ch. IV p. 118).

As before, let $V$ be a right $R G$-module. We will now consider Map $\left((E G)^{\text {left }}, V\right)$ as a cosimplicial right $R G$-module, the right $R G$-module structure being the diagonal structure relative to the right $G$-structure on $V$ and the left $G$-translation on 
$(E G)^{\text {left }}$, the cosimplicial structure being induced from the simplicial structure on $(E G)^{\text {left }}$; we recall that the diagonal structure is given by the association

$$
\operatorname{Map}\left((E G)^{\text {left }}, V\right) \times G \longrightarrow \operatorname{Map}\left((E G)^{\text {left }}, V\right), \quad(\alpha, x) \longmapsto \alpha \cdot x,
$$

where $(\alpha \cdot x) y=(\alpha(x y)) x, x \in G, y \in(E G)^{\text {left }}$; here $\alpha$ ranges over maps from $(E G)^{\text {left }}$ to $V$.

PROPOSITION 4.2. Relative to the diagonal $G$-action on $\operatorname{Map}\left((E G)^{\mathrm{left}}, V\right)$, the morphism

$$
\Phi=\left(\varphi_{0}, \ldots\right): \operatorname{Map}\left((E G)^{\text {left }}, V\right) \longrightarrow \mathbf{T}(V)
$$

of graded R-modules which, in degree $n$, is given by the association

$$
\begin{aligned}
& \varphi_{n}: \operatorname{Map}\left(G^{\times(n+1)}, V\right) \longrightarrow \operatorname{Map}\left(G^{\times(n+1)}, \square V\right), \\
& \varphi_{n}(\alpha)\left(x_{0}, \ldots, x_{n}\right)=\left(\alpha\left(x_{0}, \ldots, x_{n}\right)\right) \cdot x_{0} \cdot \ldots \cdot x_{n}, \quad x_{0}, \ldots, x_{n} \in G,
\end{aligned}
$$

where $\alpha$ ranges over maps from $G^{\times(n+1)}$ to $V$, is an isomorphism of cosimplicial right $R G$-modules.

Proof. This comes down to a tedious but straightforward verification. We leave the details to the reader.

We can now give a conceptual explanation for Proposition 2.8. The association (4.4) induces the isomorphism

$$
\Psi=\left(\psi_{0}, \psi_{1}, \ldots\right): \operatorname{Map}(E G, V) \longrightarrow \operatorname{Map}\left((E G)^{\text {left }}, V\right)
$$

of cosimplicial right $R G$-modules which, in degree $n \geq 0$, is given by the association

$$
\begin{aligned}
\psi_{n}: \operatorname{Map}\left(G^{\times(n+1)}, V\right) & \longrightarrow \operatorname{Map}\left(G^{\times(n+1)}, \square V\right) \\
\psi_{n}(\alpha)\left(x_{0}, x_{1}, \ldots, x_{n}\right) & =\alpha\left(x_{0}, x_{0} x_{1}, \ldots, x_{0} x_{1} \ldots x_{n-1}, x_{0} x_{1} \ldots x_{n}\right)
\end{aligned}
$$

where $x_{0}, x_{1}, \ldots x_{n} \in G$ and $\alpha \in \operatorname{Map}\left(G^{\times(n+1)}, V\right)$. The isomorphism $\Psi$, combined with the isomorphism $\Phi$ in Proposition 4.2, induces the association spelled out in Proposition 2.8.

We will now recall how these constructions can be formalized in the language of comonads and dual standard constructions.

Let $\mathcal{M}$ be a category, $\mathcal{L}: \mathcal{M} \rightarrow \mathcal{M}$ an endofunctor, let $\mathcal{I}$ denote the identity functor of $\mathcal{M}$, and let $c: \mathcal{L} \rightarrow \mathcal{I}$ and $\delta: \mathcal{L} \longrightarrow \mathcal{L}^{2}$ be natural transformations. Recall that the triple $(\mathcal{L}, c, \delta)$ is defined to be a comonad over $\mathcal{M}$ provided the following 
two diagrams commute:
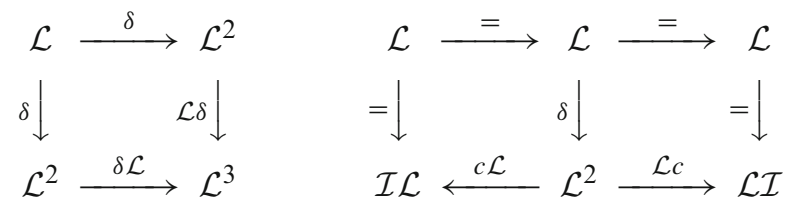

The natural transformations $c$ and $\delta$ are referred to as the counit and diagonal, respectively, of the comonad.

The standard construction associated with the comonad $(\mathcal{L}, c, \delta)$, cf. [12,35], yields the simplicial object

$$
\left(\mathcal{L}^{n+1}, d_{j}: \mathcal{L}^{n+2} \rightarrow \mathcal{L}^{n+1}, s_{j}: \mathcal{L}^{n+1} \rightarrow \mathcal{L}^{n+2}\right)_{n \in \mathbb{N}}
$$

here, for $n \geq 1$,

$$
\begin{aligned}
d_{j}^{n} & =\mathcal{L}^{j} c \mathcal{L}^{n-j}: \mathcal{L}^{n+1} \rightarrow \mathcal{L}^{n}, \quad j=0, \ldots, n, \\
s_{j}^{n} & =\mathcal{L}^{j} \delta \mathcal{L}^{n-j-1}: \mathcal{L}^{n} \rightarrow \mathcal{L}^{n+1}, \quad j=0, \ldots, n-1 .
\end{aligned}
$$

Thus, given the object $W$ of $\mathcal{M}$,

$$
\mathbf{L}(W)=\left(\mathcal{L}^{n+1}(W), d_{j}, s_{j}\right)_{n \in \mathbb{N}}
$$

is a simplicial object in $\mathcal{M}$, the standard object associated with the object $W$ and the comonad $(\mathcal{L}, c, \delta)$, where we do not distinguish in notation between the natural transformations $d_{j}$ and $s_{j}$ and the morphisms they induce after evaluation of the corresponding functors on an object; under suitable circumstances, the associated chain complex $|\mathbf{L}(W)|$ together with $c_{W}:|\mathbf{L}(W)| \rightarrow W$ is then a relatively projective resolution of $W$.

EXAMPLE 4.3. Let Set be the category of sets and ${ }_{G}$ Set that of left $G$-sets. The functor $\mathcal{V}:{ }_{G}$ Set $\rightarrow{ }_{G}$ Set which assigns to the left $G$-set $Z$ the left $G$-set $G \times Z$ endowed with diagonal $G$-action, together with the natural transformation $\gamma: \mathcal{V} \rightarrow \mathcal{V}^{2}$ which, to the left $G$-set $Z$ assigns the morphism

$$
\gamma: G \times Z \rightarrow G \times G \times Z, \quad \gamma(x, q)=(x, x, q), \quad x \in G, \quad q \in Z,
$$

and the natural transformation $\alpha: \mathcal{V} \rightarrow \mathcal{I}$ which, to the left $G$-set $Z$ assigns the morphism

$$
\alpha: G \times Z \rightarrow Z, \quad \alpha(x, q)=q, \quad x \in G, \quad q \in Z,
$$

is a comonad over the category ${ }_{G}$ Set. The standard construction associated with the comonad $(\mathcal{V}, \alpha, \gamma)$ and the $G$-set consisting of a single point is the simplicial set $E G$, that is, the homogeneous universal object associated with $G$, the simplicial set $E G$ being viewed as a principal left $G$-set. When $G$ is a Lie group, the resulting universal object $E G$ is a simplicial principal left $G$-manifold. 
An adjunction determines a comonad in the following manner [35]: Let $\mathcal{F}: \mathcal{D} \rightarrow$ $\mathcal{M}$ be a functor, suppose that the functor $\square: \mathcal{M} \rightarrow \mathcal{D}$ is right-adjoint to $\mathcal{F}$, and let

$$
\mathcal{L}=\mathcal{F} \square: \mathcal{M} \longrightarrow \mathcal{M}
$$

Let $u: \mathcal{I} \rightarrow \square \mathcal{F}$ be the unit, $c: \mathcal{L} \rightarrow \mathcal{I}$ the counit of the adjunction, and let $\delta$ be the natural transformation

$$
\delta=\mathcal{F} u \square: \mathcal{L}=\mathcal{F} \square \longrightarrow \mathcal{F} \square \mathcal{F} \square=\mathcal{L}^{2} .
$$

The data $(\mathcal{L}, c, \delta)$ constitute a comonad over the category $\mathcal{M}$.

EXAMPLE 4.4. Let $\mathcal{F}:$ Set $\rightarrow{ }_{G}$ Set be the functor which assigns to the set $Z$ the left $G$-set $G \times Z$, endowed with the obvious left $G$-action induced by left translation in $G$. This functor is left adjoint to the forgetful functor $\square:{ }_{G}$ Set $\rightarrow$ Set, and the standard construction applied to the resulting comonad and the left $G$-set $Z$ yields a simplicial set $\mathcal{E}(G, Z)$ endowed with a free left $G$-action. For $Z$ a point $o$, as a simplicial principal left $G$-set, $\mathcal{E}(G, o)$ reduces to the nonhomogeneous left universal $G$-object $(E G)^{\text {left }}$ considered earlier. When $G$ is a Lie group, the resulting left universal object $(E G)^{\text {left }}$ is a simplicial principal left $G$-manifold.

Here is a comonadic version of Proposition 2.4 above:

PROPOSITION 4.5. Given the left G-set Z, the isomorphism of left G-sets

$$
\mathcal{F} \square Z=G \times \square Z \longrightarrow G \times Z=\mathcal{V} Z, \quad(x, q) \mapsto(x, x q), \quad x \in G, \quad q \in Z,
$$

induces an isomorphism of comonads $(\mathcal{L}, c, \delta) \longrightarrow(\mathcal{V}, \alpha, \gamma)$.

This isomorphism of comonads induces the isomorphism $(E G)^{\text {left }} \rightarrow E G$, cf. (4.5) above, viewed merely as an isomorphism of simplicial principal left $G$-sets. The present identification in terms of the underlying comonads yields a conceptual explanation for this isomorphism of simplicial principal left $G$-sets. When $G$ is a Lie group, that isomorphism is one of simplicial principal left $G$-manifolds.

Remark 4.6. Let $G$ be a Lie group and let $V$ be a differentiable right $G$-module. In view of Proposition 4.1, the differentiable cosimplicial right $G$-module $\mathbf{U}(V)$ introduced as (2.18) above is simply that of smooth $V$-valued functions on the simplicial principal left $G$-manifold $E G$. Likewise, in view of Proposition 4.2, the differentiable cosimplicial right $G$-module $\mathbf{T}(V)$ introduced as $(2.5)$ above is that of smooth $V$-valued functions on the simplicial principal left $G$-manifold $(E G)^{\text {left }}$. However, the construction in the previous section shows that the attempt to introduce cohomology by taking functions on an appropriate simplicial object no longer works for a general groupoid, cf. Remark 3.4. 
In Section 6, we will introduce yet another example of a comonad which arises from an extension of Lie-Rinehart algebras. This example is crucial for the development of the equivariant de Rham cohomology relative to a general locally trivial Lie groupoid.

\section{Lie-Rinehart Equivariant Cohomology}

Let $G$ be a Lie group and $\mathfrak{g}$ its Lie algebra, and let $C \mathfrak{g}$ be the cone on $\mathfrak{g}$ in the category of differential graded Lie algebras. Thus momentarily we are working over the reals as ground ring. Given a smooth manifold $M$ and a smooth action of $G$ on $M$, the infinitesimal $\mathfrak{g}$-action $\mathfrak{g} \rightarrow \operatorname{Vect}(M)$ on $M$ induces a canonical (differential graded) $C \mathfrak{g}$-module structure on the de Rham algebra $\mathcal{A}(M)$ of $M$ via the operations of contraction and Lie derivative. In [29], we established a close relationship between the $G$-equivariant de Rham theory of $M$ and the relative $\operatorname{Ext}_{(C \mathfrak{g}, \mathfrak{g})}(\mathbb{R}, \mathcal{A}(M)$ ) (cf. Example 2.10 above) where the term relative is used in the sense of [18]. In particular, when $G$ is compact, the invariants $\operatorname{Ext}_{(C \mathfrak{g}, \mathfrak{g})}(\mathbb{R}, \mathcal{A}(M))^{\pi_{0}(G)}$ relative to the group $\pi_{0}(G)$ of connected components of $G$ coincide with the $G$-equivariant de Rham cohomology of $M$, cf. [29]. The question we will explore now is whether that relative derived Ext has a meaning for a general Lie-Rinehart algebra and, if so, what it then signifies.

Let $R$ be an arbitrary commutative ring with 1 and $\mathfrak{g}$ an $R$-Lie algebra, which we suppose to be projective as an $R$-module. In [29], we defined the relative differential $\operatorname{Ext}_{(C \mathfrak{g}, \mathfrak{g})}$ in terms of a suitable monad; we have reproduced the details in Example 2.10 above. Since, over the reals, when $G$ is a compact and connected Lie group, $\operatorname{Ext}_{(C \mathfrak{g}, \mathfrak{g})}(\mathbb{R}, \mathcal{A}(M))$ coincides with the $G$-equivariant de Rham cohomology of $M$, we refer to $\operatorname{Ext}_{(C \mathfrak{g}, \mathfrak{g})}(R, \cdot)$ as the infinitesimal equivariant cohomology relative to $\mathfrak{g}$. In [29], we also spelled out a suitable comonad which, in turn, leads to the corresponding relative bar resolution, where the term "relative" is intended to hint at the fact that this is the bar resolution for the relative situation under discussion, cf. e.g., [34] (Ch. 9 and Ch. 10). Since U[C $\mathrm{g}]$ is actually a Hopf algebra, a homogeneous version of the corresponding relative bar resolution is available as well; this homogeneous relative bar resolution leads to the simplicial Weil coalgebra explored in [29]. The construction of the simplicial Weil coalgebra does not extend to a general Lie-Rinehart algebra. However, the construction of the dual object, the CCE algebra or, equivalently, Maurer-Cartan algebra, cf. [42] for this terminology, extends to a general Lie-Rinehart algebra. This leads to a notion of Lie-Rinehart equivariant cohomology. We will now explain the details.

We begin with some preparations. Let $\mathfrak{g}$ and $\mathfrak{g}^{\prime}$ be ordinary Lie algebras over $R$, and let $\Phi: \mathfrak{g} \rightarrow \mathfrak{g}^{\prime}$ be a morphism of Lie algebras. Let $N$ be a left $\mathfrak{g}$-module, $N^{\prime}$ a left $\mathfrak{g}^{\prime}$-module, view $N^{\prime}$ as a left $\mathfrak{g}$-module via $\Phi$, and let $\varphi: N^{\prime} \rightarrow N$ be a morphism of $R$-modules. Recall that the pair $(\varphi, \Phi)$ is referred to as a morphism of actions $(N, \mathfrak{g}) \rightarrow\left(N^{\prime}, \mathfrak{g}^{\prime}\right)$ provided $\varphi$ is a morphism of $\mathfrak{g}$-modules. We will use the notation $\operatorname{Alt}(\mathfrak{g}, N)$, etc. for the ordinary graded $R$-module or chain complex of 
$N$-valued alternating forms on $\mathfrak{g}$. It is a classical fact (and entirely obvious) that a morphism of actions $(N, \mathfrak{g}) \rightarrow\left(N^{\prime}, \mathfrak{g}^{\prime}\right)$ induces the morphism

$$
\operatorname{Alt}\left(\mathfrak{g}^{\prime}, N^{\prime}\right) \stackrel{\Phi^{*}}{\longrightarrow} \operatorname{Alt}\left(\mathfrak{g}, N^{\prime}\right) \stackrel{\varphi_{*}}{\longrightarrow} \operatorname{Alt}(\mathfrak{g}, N)
$$

between the CCE complexes and hence, when $\mathfrak{g}$ and $\mathfrak{g}^{\prime}$ are projective as modules over $R$, the morphism

$$
\mathrm{H}^{*}\left(\mathfrak{g}^{\prime}, N^{\prime}\right) \longrightarrow \mathrm{H}^{*}(\mathfrak{g}, N)
$$

on cohomology. The morphism (5.1) can also be written as the composite morphism

$$
\operatorname{Alt}\left(\mathfrak{g}^{\prime}, N^{\prime}\right) \stackrel{\varphi_{*}}{\longrightarrow} \operatorname{Alt}\left(\mathfrak{g}^{\prime}, N\right) \stackrel{\Phi^{*}}{\longrightarrow} \operatorname{Alt}(\mathfrak{g}, N)
$$

of graded $R$-modules but the graded $R$-module $\operatorname{Alt}\left(\mathfrak{g}^{\prime}, N\right)$ does not in general acquire a differential compatible with the other structure since only $\mathfrak{g}$ is supposed to act on $N$, not $\mathfrak{g}^{\prime}$.

Adapting the notion of morphism of actions to Lie-Rinehart algebras via the description (5.2) leads to the notion of comorphism of Lie-Rinehart algebras, introduced in [16]. A comorphism is not the dual of a morphism, though. The corresponding notion for Lie algebroids is that of morphism of Lie algebroids and goes back to [1]. Thus, let $(A, L)$ and $\left(A^{\prime}, L^{\prime}\right)$ be Lie-Rinehart algebras. A comorphism $(\varphi, \Phi):(A, L) \rightarrow\left(A^{\prime}, L^{\prime}\right)$ of Lie-Rinehart algebras consists of a morphism $\varphi: A^{\prime} \rightarrow A$ of algebras and a morphism $\Phi: L \rightarrow A \otimes_{A^{\prime}} L^{\prime}$ of $A$-modules such that (i) and (ii) below are satisfied:

(i) The diagram

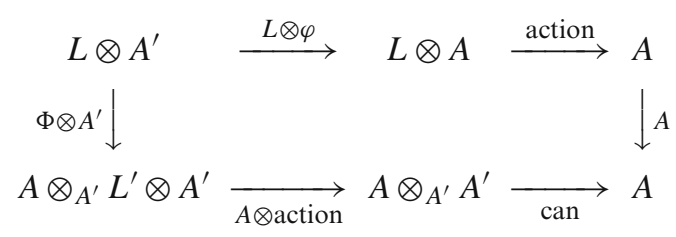

is commutative.

(ii) Given $\alpha_{1}, \alpha_{2} \in L$ with $\Phi\left(\alpha_{1}\right)=\sum a_{i}^{1} \beta_{i}^{1}$ and $\Phi\left(\alpha_{2}\right)=\sum a_{j}^{2} \beta_{j}^{2}$,

$$
\Phi\left(\left[\alpha_{1}, \alpha_{2}\right]\right)=\sum a_{i}^{1} a_{j}^{2} \otimes\left[\beta_{i}^{1}, \beta_{j}^{2}\right]+\sum \alpha_{1}\left(a_{j}^{2}\right) \otimes \beta_{j}^{2}-\sum \alpha_{2}\left(a_{i}^{1}\right) \otimes \beta_{i}^{1} .
$$

It is not required that $\Phi$ lift to a morphism $L \rightarrow L^{\prime}$ of $R$-modules or even $R$-Lie algebras.

EXAMPLE. A smooth map $f: M \rightarrow N$ between smooth manifolds induces the comorphism

$$
\left(f^{*}, f_{*}\right):\left(C^{\infty}(M), \operatorname{Vect}(M)\right) \longrightarrow\left(C^{\infty}(N), \operatorname{Vect}(N)\right)
$$


of Lie-Rinehart algebras in an obvious manner; in particular $f^{*}: C^{\infty}(N) \rightarrow$ $C^{\infty}(M)$ is the induced morphism of commutative algebras.

A comorphism $(\varphi, \Phi):(A, L) \rightarrow\left(A^{\prime}, L^{\prime}\right)$ of Lie-Rinehart algebras induces the morphism

$$
\operatorname{Alt}_{A^{\prime}}\left(L^{\prime}, A^{\prime}\right) \stackrel{\varphi_{*}}{\longrightarrow} \operatorname{Alt}_{A}\left(A \otimes_{A^{\prime}} L^{\prime}, A\right) \stackrel{\Phi^{*}}{\longrightarrow} \operatorname{Alt}_{A}(L, A)
$$

of Maurer-Cartan algebras. As a morphism of graded $A^{\prime}$-algebras, this morphism is well defined in view of (i). The requirement (ii) entails the compatibility with the differentials.

Let $(A, L)$ be a Lie-Rinehart algebra. The cone $C L$ in the category of differential graded $R$-Lie algebras has no obvious meaning as a differential graded LieRinehart algebra. Indeed, this cone contains the suspended object $s L$ as a graded Lie ideal but not as a differential graded Lie ideal, and the injection of $L$ into $C L$ does not induce any kind of $(C L)$-action on $A$ whatsoever.

On the other hand, the Lie-Rinehart axioms imply that the (differential graded) action

$$
C L \otimes \operatorname{Alt}_{R}(L, M) \longrightarrow \operatorname{Alt}_{R}(L, M)
$$

passes to an action

$$
C L \otimes \operatorname{Alt}_{A}(L, M) \longrightarrow \operatorname{Alt}_{A}(L, M)
$$

of $C L$ on $\operatorname{Alt}_{A}(L, M)$ which is in fact a differential graded $(C L)$-action on $\operatorname{Alt}_{A}(L, M)$. Since the operation of contraction is compatible with the $A$-module structure in the sense that $i_{a \alpha}=a i_{\alpha}$ for $a \in A$ and $\alpha \in L$, the operation $\lambda$ of Lie derivative necessarily satisfies the familiar identity

$$
\lambda_{a \alpha}(\omega)=a \lambda_{\alpha}(\omega)+d a \cup i_{\alpha}(\omega) \quad\left(a \in A, \quad \alpha \in L, \omega \in \operatorname{Alt}_{A}(L, M)\right) .
$$

In particular, relative to the operation of Lie-derivative, $\operatorname{Alt}_{A}(L, M)$ is just an $L$-module, not an $(A, L)$-module.

Let $\mathcal{N}$ be a (differential graded) $\operatorname{Alt}_{A}(L, A)$-module which is, furthermore, endowed with a $(C L)$-module structure; for intelligibility we will write the $(C L)$ module structure on $\mathcal{N}$ in terms of the familiar notation $\lambda$ of Lie-derivative and $i$ of contraction. Abstracting from the property just isolated, we will refer to $\mathcal{N}$ as an $(A, C L)$-module whenever the identity (5.8) is satisfied, with $\mathcal{N}$ substituted for $\operatorname{Alt}_{A}(L, M)$.

Consider the differential graded algebra $\mathrm{U}_{A}[C L]$ of operators on $\operatorname{Alt}_{A}(L, A)$ generated by $A$ and $C L$. In this algebra, given $a \in A$ and $\alpha \in L$, the operators $\lambda_{a \alpha}$, $\lambda_{\alpha}$ and $i_{\alpha}$ are related by the identity

$$
\lambda_{a \alpha}-a \lambda_{\alpha}=(d a) i_{\alpha}
$$


In order for this identity to make sense we must extend the coefficients from $A$ to $\operatorname{Alt}_{A}(L, A)$. Thus, consider the crossed product algebra

$$
\mathrm{U}[L] \odot \operatorname{Alt}_{A}\left(L, \Lambda_{A}[s L]\right)
$$

requiring that the identity (5.9) be satisfied leads us to the quotient algebra such that this identity holds. Consequently, the differential graded algebra $\mathrm{U}_{A}[C L]$ of operators on $\mathrm{Alt}_{A}(L, A)$ generated by $A$ and $C L$ is a certain quotient of that algebra.

For an ordinary Lie algebra $\mathfrak{g}$, in [29], we introduced the simplicial Weil coalgebra of $\mathfrak{g}$ to be the simplicial CCE coalgebra $\Lambda_{\partial}^{\prime}[s E \mathfrak{g}]$ of the total simplicial object $E \mathfrak{g}$ associated with $\mathfrak{g}$. For reasons explained in [23], the construction of the CCE coalgebra does not extend to a general Lie-Rinehart algebra. However the dual object, the CCE algebra or Maurer-Cartan algebra, extends; in fact, this is just the differential graded commutative algebra $\operatorname{Alt}_{A}(L, A)$ associated with the Lie-Rinehart algebra $(A, L)$. On the symmetric monoidal category of differential graded cocommutative coalgebras over $R$, the total object functor associates with any differential graded cocommutative coalgebra $C$ the simplicial differential graded coalgebra $E C$ whose condensed object $|E C|^{\bullet}$ is a contractible differential graded coalgebra in the sense that the counit $|E C|^{\bullet} \rightarrow R$ is a chain equivalence.

On the symmetric monoidal category of differential graded commutative algebras over $R$, the construction of the total simplicial object dualizes to that of a total cosimplicial differential graded algebra functor $\mathcal{C}$; this functor assigns to the differential graded commutative algebra $\mathcal{A}$ the differential graded commutative algebra $\mathcal{C} \mathcal{A}$ having, for $n \geq 0$, the tensor power $(\mathcal{C A})_{n}=\mathcal{A}^{\otimes(n+1)}$ over the ground ring $R$ of $n+1$ copies of $\mathcal{A}$ as degree $n$ constituent, with coface and codegeneracy operators being induced by the multiplication map $\mu: \mathcal{A} \otimes \mathcal{A} \rightarrow \mathcal{A}$ of $\mathcal{A}$ and the unit map $u: R \rightarrow A$ of $A$. Totalization and normalization yield the differential graded commutative algebra

$$
|\mathcal{C} \mathcal{A}|^{\bullet}
$$

which is a contractible differential graded algebra in the sense that the unit $R \rightarrow$ $|\mathcal{C} \mathcal{A}|^{\bullet}$ is a chain equivalence.

This construction applies, in particular, to the differential graded $R$-algebra $\mathcal{A}=$ $\operatorname{Alt}_{A}(L, A)$ but, beware, the tensor powers are taken over the ground ring $R$ (and not over $A$; indeed the construction would not then be well defined). Furthermore, $C L$ being an ordinary differential graded $R$-Lie algebra, the $(C L)$-action on $\operatorname{Alt}_{A}(L, A)$ extends to a $(C L)$-action on $\mathcal{C} \operatorname{Alt}_{A}(L, A)$ and hence on $\left|\mathcal{C} \operatorname{Alt}_{A}(L, A)\right|^{\bullet}$, and the invariants $\left(\left|\mathcal{C} \operatorname{Alt}_{A}(L, A)\right|^{\bullet}\right)^{C L}$ constitute a differential graded $R$-subalgebra.

For illustration, consider the special case where $A$ coincides with the ground ring and where $L$ is an ordinary Lie algebra $\mathfrak{g}$. Then $\left(|\mathcal{C A l t} A(L, A)|^{\bullet}\right)^{C L}$ reduces to 
$\left(|\mathcal{C} \operatorname{Alt}(\mathfrak{g}, R)|^{\bullet}\right)^{C \mathfrak{g}}$, and $\mathcal{C} \operatorname{Alt}(\mathfrak{g}, R)$ is canonically isomorphic to

$$
\operatorname{Hom}\left(\Lambda_{\partial}^{\prime}[s E \mathfrak{g}], R\right) \cong \operatorname{Hom}\left(E \Lambda_{\partial}^{\prime}[s \mathfrak{g}], R\right)
$$

whence

$$
\mathrm{H}\left(\left(|\mathcal{C} \operatorname{Alt}(\mathfrak{g}, R)|^{\bullet}\right)^{C \mathfrak{g}}\right) \cong \operatorname{Ext}_{(C \mathfrak{g}, \mathfrak{g})}(R, R) .
$$

In particular, when $\mathfrak{g}$ is reductive, $H^{*}(\mathfrak{g})$ is the exterior Hopf algebra $\Lambda[\operatorname{Prim}(\mathfrak{g})]$ on its primitives $\operatorname{Prim}(\mathfrak{g}) \subseteq H^{*}(\mathfrak{g})$, and

$$
\operatorname{Ext}_{(C \mathfrak{g}, \mathfrak{g})}(R, R) \cong \mathrm{S}\left[s^{-1} \operatorname{Prim}(\mathfrak{g})\right],
$$

the symmetric algebra on the desuspension $s^{-1} \operatorname{Prim}(\mathfrak{g})$ of the primitives $\operatorname{Prim}(\mathfrak{g}) \subseteq \mathrm{H}^{*}(\mathfrak{g})$.

Slightly more generally, suppose that $\mathfrak{g}$ acts on the commutative algebra $A$ by derivations, and let $L=A \odot \mathfrak{g}$ be the resulting crossed product $(R, A)$-Lie algebra, cf. e.g., [20]. Then the differential graded algebra $\operatorname{Alt}_{A}(L, A)$ reduces to $\operatorname{Alt}(\mathfrak{g}, A)$ and $\mathcal{C} \operatorname{Alt}_{A}(L, A)$ is canonically isomorphic to $\mathcal{C A l t}(\mathfrak{g}, A)$ or

$$
\operatorname{Hom}\left(\Lambda_{\partial}^{\prime}[s E \mathfrak{g}], A\right) \cong \operatorname{Hom}\left(E \Lambda_{\partial}^{\prime}[s \mathfrak{g}], A\right) .
$$

When $\mathfrak{g}$ is reductive, $\mathrm{H}^{*}(\mathfrak{g}, A) \cong \Lambda[\operatorname{Prim}(\mathfrak{g})] \otimes A^{\mathfrak{g}}$, and

$$
\mathrm{H}\left(\left(|\mathcal{C} \operatorname{Alt}(\mathfrak{g}, A)|^{\bullet}\right)^{C \mathfrak{g}}\right) \cong \mathrm{S}\left[s^{-1} \operatorname{Prim}(\mathfrak{g})\right] \otimes A^{\mathfrak{g}} .
$$

To unravel the structure, we note that, relative to the obvious structures, $(A \otimes A, L \otimes A)$ and $(A \otimes A, A \otimes L)$ acquire Lie-Rinehart structures, and the direct sum

$$
L^{\times}=L \otimes A \oplus A \otimes L
$$

of $(A \otimes A)$-modules acquires an obvious $(R, A \otimes A)$-Lie algebra structure. We use the notation $L^{\times}$since $\left(A \otimes A, L^{\times}\right)$is the categorical product of $(A, L)$ with itself in the category of Lie-Rinehart algebras, and we will therefore occasionally write

$$
(A, L) \times(A, L)=\left(A \otimes A, L^{\times}\right) .
$$

The corresponding product construction for Lie algebroids was introduced in [1]. A special case of the construction in [1] is the following: Over the reals $\mathbb{R}$ as ground ring, when $L$ is the $\left(\mathbb{R}, C^{\infty}(M)\right)$-Lie algebra of smooth vector fields on the smooth manifold $M$, when the ordinary tensor product is replaced with a suitably completed tensor product (Frechét tensor product), (5.14) is the familiar decomposition of the Lie algebra of smooth vector fields on the product $M \times M$ into two summands corresponding to the two factors $M$. Accordingly

$$
\operatorname{Alt}_{A \otimes A}\left(L^{\times}, A \otimes A\right) \cong \operatorname{Alt}_{A}(L, A) \otimes \operatorname{Alt}_{A}(L, A)
$$


canonically. The ordinary diagonal morphism

$$
\Delta: L \longrightarrow L \oplus L \cong A \otimes_{A \otimes A} L^{\times}, \Delta(\rho)=(\rho, \rho)(\rho \in L)
$$

of $A$-modules and the multiplication map $\mu: A \otimes A \rightarrow A$ constitute a comorphism

$$
(\mu, \Delta):(A, L) \longrightarrow\left(A \otimes A, L^{\times}\right)=(A, L) \times(A, L)
$$

of Lie-Rinehart algebras, the Lie-Rinehart diagonal map for $(A, L)$. In this case, the defining condition (5.3) of a comorphism simply comes down to the fact that $L$ acts on $A$ by derivations and the defining condition (5.4) is plainly satisfied.

The induced morphism of Maurer-Cartan algebras of the kind (5.5) coincides with the multiplication map of $\operatorname{Alt}_{A}(L, A)$.

With the notion of comorphism as morphism, Lie-Rinehart algebras constitute a symmetric monoidal category, and the Lie-Rinehart diagonal is a diagonal morphism in this category. Consequently, given the Lie-Rinehart algebra $(A, L)$, the total object

$$
E(A, L)=(E A, E L)
$$

is defined as a simplicial Lie-Rinehart algebra. Thus $E L$ is a simplicial $R$-Lie algebra, $E A$ is a cosimplicial $R$-algebra, and the structure is encoded in two pairings

$$
E A \otimes E L \longrightarrow E L, E L \otimes E A \longrightarrow E A
$$

satisfying the appropriate Lie-Rinehart axioms.

Application of the Maurer-Cartan functor $\operatorname{Alt}_{(\cdot)}(\cdot, \cdot)$ to the simplicial LieRinehart algebra $(E A, E L)$ yields the cosimplicial differential graded algebra

$$
\operatorname{Alt}_{E A}(E L, E A) \text {. }
$$

However, this is precisely the cosimplicial differential graded algebra $\mathcal{C} \operatorname{Alt}_{A}(L, A)$ considered earlier, and we will refer to it as the cosimplicial Maurer-Cartan algebra associated with the simplicial Lie-Rinehart algebra (EA,EL). Furthermore, the $(C L)$-action on $\operatorname{Alt}_{A}(L, A)$ by contraction and Lie-derivative extends canonically to a $(C L)$-action on $\operatorname{Alt}_{E A}(E L, E A)$ compatible with the cosimplicial structure in the sense that each cosimplicial operator is compatible with the $(C L)$-actions.

Let $\mathcal{N}$ be an $(A, C L)$-module and view it as the trivial cosimplicial $(A, C L)$ module. The product $(E A) \otimes \mathcal{N}$ of cosimplicial objects is defined and inherits a canonical $(A, C L)$-module structure compatible with the cosimplicial structure. Application of the Maurer-Cartan functor $\operatorname{Alt}_{(\cdot)}(\cdot, \cdot)$ to the simplicial Lie-Rinehart algebra $(E A, E L)$ yields the cosimplicial differential graded $\operatorname{Alt}_{E A}(E L, E A)$-module

$$
\operatorname{Alt}_{E A}(E L,(E A) \otimes \mathcal{N}) .
$$


We define the $(A, L)$-equivariant cohomology $\mathrm{H}_{(A, L)}^{*}(\mathcal{N})$ of $\mathcal{N}$ to be the homology

$$
\mathrm{H}_{(A, L)}^{*}(\mathcal{N})=\mathrm{H}\left(\operatorname{Alt}_{E A}(E L,(E A) \otimes \mathcal{N})^{C L}\right) .
$$

We can view this theory also as the relative

$$
\operatorname{Ext}_{(A, C L, L)}(E A, \mathcal{N}) .
$$

ILLUSTRATION. Let $\mathcal{F}$ be a foliation of the smooth manifold $M$, let $A=$ $C^{\infty}(M)$, let $\tau_{\mathcal{F}}$ be the tangent bundle of $\mathcal{F}$, and let $L$ be the $(\mathbb{R}, A)$-Lie algebra of smooth vector fields tangent to $\mathcal{F}$. Thus the injection $L \rightarrow L_{M}=\operatorname{Vect}(M)$ is a morphism of $(\mathbb{R}, A)$-Lie algebras, and the de Rham complex $\mathcal{N}=\mathcal{A}(M)=\operatorname{Alt}_{A}\left(L_{M}, A\right)$ of $M$ is an $(A, L, C L)$-module via the $A$-module structure and the operations of contraction and Lie derivative. Under these circumstances, $\mathrm{H}_{(A, L)}^{*}(\mathcal{N})$ is the de Rham cohomology of $M$ that is equivariant relative to the foliation $\mathcal{F}$.

\section{Extensions of Lie-Rinehart Algebras}

The algebraic analog of an "Atiyah sequence" or of a "transitive Lie algebroid" (see below for details) is an extension of Lie-Rinehart algebras [24].

Let $L^{\prime}, L, L^{\prime \prime}$ be $(R, A)$-Lie algebras. An extension of $(R, A)$-Lie algebras is defined to be a short exact sequence

$$
\text { e: } 0 \longrightarrow L^{\prime} \longrightarrow L \stackrel{p}{\longrightarrow} L^{\prime \prime} \longrightarrow 0
$$

in the category of $(R, A)$-Lie algebras; notice in particular that the Lie algebra $L^{\prime}$ necessarily acts trivially on $A$.

EXAMPLE 6.1. Let the ground ring $R$ be the field $\mathbb{R}$ of real numbers, let $B$ be a smooth finite dimensional manifold, let $A$ be the algebra of smooth functions on $B$, let $G$ be a Lie group, and let $\xi: P \rightarrow B$ be a principal right $G$-bundle. The vertical subbundle $\mathrm{T} P_{\text {vert }} \rightarrow P$ of the tangent bundle $\tau_{P}: \mathrm{T} P \rightarrow B$ of $P$ is well known to be trivial (beware, not equivariantly trivial), having as fibre the Lie algebra $\mathfrak{g}$ of $G$, that is, $\mathrm{T} P_{\text {vert }} \cong P \times \mathfrak{g}$. Dividing out the $G$-actions, we obtain an extension

$$
0 \longrightarrow \operatorname{ad}(\xi) \longrightarrow \tau_{P} / G \longrightarrow \tau_{B} \longrightarrow 0
$$

of vector bundles over $B$, where $\tau_{B}$ is the tangent bundle of $B$. This sequence has been introduced by Atiyah [3] and is now usually referred to as the Atiyah sequence of the principal bundle $\xi$; here ad $(\xi): P \times_{G} \mathfrak{g} \longrightarrow B$ is the bundle associated with the principal bundle and the adjoint representation of $G$ on its Lie algebra $\mathfrak{g}$. The spaces $\mathfrak{g}(\xi)=\Gamma \operatorname{ad}(\xi)$ and $\mathrm{E}(\xi)=\Gamma\left(\tau_{P} / G\right) \cong \Gamma\left(\tau_{P}\right)^{G}$ of smooth sections inherit obvious Lie algebra structures, in fact $(\mathbb{R}, A)$-Lie algebra structures, and

$$
0 \longrightarrow \mathfrak{g}(\xi) \longrightarrow \mathrm{E}(\xi) \longrightarrow \operatorname{Vect}(B) \longrightarrow 0
$$


is an extension of $(\mathbb{R}, A)$-Lie algebras; here $\operatorname{Vect}(B)$ is the $(\mathbb{R}, A)$-Lie algebra of smooth vector fields on $B$, and $\mathfrak{g}(\xi)$ is well known to be, in an obvious way, the Lie algebra of the group of gange transformations of $\xi$, see Section 7 below. The resulting Lie algebroid

$$
\tau_{P} / G:(\mathrm{T} P) / G \longrightarrow B
$$

over $B$ is plainly transitive in the sense that the "anchor" $\mathrm{E}(\xi) \rightarrow \operatorname{Vect}(B)$ is surjective. We will refer to this Lie algebroid as the Atiyah algebroid of the principal bundle $\xi$.

Over a general ground ring $R$, consider an extension

$$
\text { e: } 0 \longrightarrow L^{\prime} \longrightarrow L \longrightarrow L^{\prime \prime} \longrightarrow 0
$$

of $(R, A)$-Lie algebras of the kind (6.1). To avoid unnecessary complications, we will suppose that $L^{\prime}, L$, and $L^{\prime \prime}$ are finitely generated and projective as $A$-modules. Since $L^{\prime}$ is an ordinary $A$-Lie algebra that acts trivially on $A$, unlike the situation in Section 5 above, the cone $C^{\mathbf{e}} L=\left(s L^{\prime}\right) \rtimes L$ in the category of differential graded $(R, A)$-Lie algebras makes perfect sense as the differential graded Lie-Rinehart algebra $\left(A, C^{\mathbf{e}} L\right)$; more precisely, the projection from $L$ to $L^{\prime \prime}$ extends to a morphism $C^{\mathrm{e}} L \rightarrow L^{\prime \prime}$ of differential graded Lie algebras and, in this way, the pair $\left(A, C^{\mathbf{e}} L\right)$ acquires a differential graded Lie-Rinehart algebra structure in such a way that

$$
\left(A, C^{\mathbf{e}} L\right) \longrightarrow\left(A, L^{\prime \prime}\right)
$$

is a morphism of differential graded Lie-Rinehart algebras. We note that, by construction, the cone $C^{\mathbf{e}} L$ contains the suspended object $s L^{\prime}$ as a graded Lie ideal but not as a differential graded Lie ideal since the values of the differential, applied to elements of $s L^{\prime}$, lie in $L$. The notation $C^{\mathrm{e}}$ is intended to indicate a cone construction, whence the letter $C$, but relative the extension $\mathbf{e}$, whence the superscript.

The notion of $\left(A, C^{\mathbf{e}} L\right)$-module is defined in the obvious way and, working formally as in Section 5 above, we introduce the relative

$$
\operatorname{Ext}_{\left(A, C^{\mathrm{e}} L, L\right)}
$$

via the appropriate comonad as follows:

Let $\mathrm{U}_{A}[L]$ be the universal algebra associated with the Lie-Rinehart algebra $(A, L)$ and $\mathrm{U}_{A}\left[C^{\mathbf{e}} L\right]$ the universal differential graded algebra associated with the differential graded Lie-Rinehart algebra $\left(A, C^{\mathbf{e}} L\right)$. Consider the pair $(\mathcal{R}, \mathcal{S})=$ $\left(\mathrm{U}_{A}\left[C^{\mathbf{e}} L\right], \mathrm{U}_{A}[L]\right)$, with $\mathcal{R} \supseteq \mathcal{S}$. This pair gives rise to a resolvent pair of categories, cf. [34] (IX.6), that is to say, the functor

$$
\mathcal{F}: \operatorname{Mod}_{\mathcal{S}} \longrightarrow \operatorname{Mod}_{\mathcal{R}}
$$


which assigns to the right $\mathcal{S}$-module $N$ the induced $\mathcal{R}$-module $\mathcal{F}(N)=N \otimes_{\mathcal{S}} \mathcal{R}$ is left adjoint to the forgetful functor $\square: \operatorname{Mod}_{\mathcal{R}} \rightarrow \operatorname{Mod}_{\mathcal{S}}$. Relative (co)homology is then defined and calculated in terms of a relatively projective resolution in the sense of [18]. Given the right $\mathcal{R}$-module $N$, the standard construction $\mathbf{L}(N)$ arising from $N$ and the comonad $(\mathcal{L}, c, \delta)$ associated with the adjunction is a simplicial object whose associated chain complex $|\mathbf{L}(N)|$ coincides with the standard relatively projective resolution of $N$ in the sense of [18].

Let $\left(\Lambda_{A}^{\prime}\right)_{\partial}\left[s L^{\prime}\right]$ be the CCE coalgebra of the $A$-Lie algebra $L^{\prime}$, taken in the category of $A$-modules - cf. the discussion of the simplicial Weil coalgebra in the previous section as well as the more detailed description of the CCE coalgebra in [29]. Furthermore, let $\mathcal{C}_{(A, L)}$ be the category of chain complexes in the category of right $(A, L)$-modules and let

$$
\mathcal{F}: \mathcal{C}_{(A, L)} \longrightarrow \operatorname{Mod}_{\left(A, C^{\mathrm{e}} L\right)}
$$

be the functor which assigns to the right $(A, L)$-chain complex $N$ the totalized CCE complex

$$
\mathcal{F} N=N \otimes_{(A, L)} \mathrm{U}_{A}\left[C^{\mathbf{e}} L\right] \cong N \otimes_{\left(A, \tau_{L^{\prime}}\right)}\left(\Lambda_{A}^{\prime}\right)_{\partial}\left[s L^{\prime}\right]
$$

calculating the Lie algebra homology of the $A$-Lie algebra $L^{\prime}$ with coefficients in $N$, viewed as an $\left(A, L^{\prime}\right)$-module (suitably interpreted relative to the chain complex structure on $N)$. Here the notation $\cdot \otimes_{\left(A, \tau_{L^{\prime}}\right)}$ - refers to the twisted tensor product relative to the universal twisting cochain $\tau_{L^{\prime}}:\left(\Lambda_{A}^{\prime}\right)_{\partial}\left[s L^{\prime}\right] \longrightarrow \mathrm{U}_{A}\left[L^{\prime}\right]$ in the category of $A$-modules. Now, on the category of $\left(A, C^{\mathbf{e}} L\right)$-modules, the differential functors $\operatorname{Tor}^{\left(A, C^{\mathrm{e}} L, L\right)}$ and $\operatorname{Ext}_{\left(A, C^{\mathrm{e}} L, L\right)}$ are defined: Given the right $\left(A, C^{\mathrm{e}} L\right)$-module $\mathbf{W}$ and the left $\left(A, C^{\mathbf{e}} L\right)$-module $\mathbf{U}$, the relative differential $\operatorname{Tor}^{\left(A, C^{\mathrm{e}} L, L\right)}(\mathbf{W}, \mathbf{U})$ is the homology of the chain complex

$$
|\mathbf{L}(\mathbf{W})| \otimes_{\left(A, C^{\mathrm{e}} L\right)} \mathbf{U} .
$$

Given the right $\left(A, C^{\mathbf{e}} L\right)$-modules $\mathbf{W}$ and $\mathbf{V}$, the relative differential $\operatorname{Ext}_{\left(A, C^{\mathrm{e}} L, L\right)}$ $(\mathbf{W}, \mathbf{V})$ is the homology of the chain complex

$$
\operatorname{Hom}_{\left(A, C^{\mathbf{e}} L\right)}(|\mathbf{L}(\mathbf{W})|, \mathbf{V}) \text {. }
$$

\section{The Cone on the Lie Algebroid Associated with a Lie Groupoid}

Let $\Omega$ be a Lie groupoid and let $\tau_{\Omega}^{t}: \mathrm{T}^{t} \Omega \rightarrow \Omega$ be the tangent bundle of the foliation given by the $t$-fibers. Recall that, as a smooth vector bundle, the Lie algebroid $\lambda_{\Omega}: A \Omega \rightarrow B_{\Omega}$ associated with $\Omega$ is the pull back vector bundle $1^{*} \tau_{\Omega}^{t}$ across the object inclusion map 1: $B_{\Omega} \rightarrow \Omega$ of $\tau_{\Omega}^{t}$. Since the left-invariant vector fields on $\Omega$ are closed under the Lie bracket, the space $\Gamma\left(\lambda_{\Omega}\right)$ of sections is well known to acquire a Lie bracket, the Lie algebroid bracket. Furthermore, the anchor $a_{\Omega}: \lambda_{\Omega} \rightarrow$ $\tau_{B_{\Omega}}$ is the vector bundle morphism which maps each section $X$ of $\lambda_{\Omega}$ to the $s$-projection of the corresponding left-invariant vector field. The Lie algebroid associated 
with $\Omega$ is also the Atiyah algebroid of an associated principal bundle $\xi: P \rightarrow B_{\Omega}$ having $\Omega$ as its gauge groupoid. We will write the resulting Lie-Rinehart algebra as

$$
\left(A_{B_{\Omega}}, L_{\Omega}\right)=\left(C^{\infty}\left(B_{\Omega}\right), \Gamma\left(\lambda_{\Omega}\right)\right) .
$$

The anchor $a_{\Omega}$ amounts to a morphism of Lie-Rinehart algebras from $\left(A_{B_{\Omega}}, L_{\Omega}\right)$ to $\left(A_{B_{\Omega}}, \operatorname{Vect}\left(B_{\Omega}\right)\right)$.

Let $\theta: E \rightarrow B_{\Omega}$ be a vector bundle. Recall that a left $\Omega$-module structure on $\theta$ induces a left $\left(A_{B_{\Omega}}, L_{\Omega}\right)$-module structure on the $A_{B_{\Omega}}$-module $\Gamma(\theta)$ of sections of $\theta$. Indeed, let $G$ be a Lie group, let $\xi: P \rightarrow B_{\Omega}$ be a principal right $G$-bundle having $\Omega$ as its gauge groupoid and such that $\theta$ is the vector bundle associated with $\xi$ and the left $G$-representation $V$. Then $L_{\Omega}=\Gamma\left(\lambda_{\Omega}\right)$ can be taken to be the space of $G$-equivariant sections of the tangent bundle $\tau_{p}$ : T $P \longrightarrow P$ of $P$, the space $\Gamma(\theta)$ of sections of $\theta$ arises as the space of smooth $G$-equivariant maps from $P$ to $V$, and the $L_{\Omega}$-action on that space of maps is the obvious one through $G$-equivariant vector fields on $P$. See also Theorem 4.1.6 in [31] (p. 152), where this kind of structure is referred to as an infinitesimal action.

Since the Lie algebroid $\lambda_{\Omega}$ of $\Omega$ is the Atiyah algebroid of an associated principal $G$-bundle $\xi: P \rightarrow B_{\Omega}$ on $B_{\Omega}$ (such that $\Omega$ is the gauge groupoid of $\xi$ ), the corresponding extension (6.3) of ( $\mathbb{R}, A_{B_{\Omega}}$ )-Lie algebras arising from the Atiyah sequence (6.2) of $\xi$ is available; this extension takes the form

$$
\mathbf{e}_{\xi}: 0 \longrightarrow \mathfrak{g}(\xi) \longrightarrow \mathrm{E}(\xi) \longrightarrow \operatorname{Vect}\left(B_{\Omega}\right) \longrightarrow 0 .
$$

Now the cone $C^{\mathbf{e}_{\xi}} L_{\Omega}$ introduced in Section 6 is defined, and $\left(A_{B_{\Omega}}, C^{\mathbf{e}_{\xi}} L_{\Omega}\right)$ is a differential graded Lie-Rinehart algebra. We will henceforth write $C^{\Omega} L_{\Omega}$ rather than $C^{\mathbf{e}_{\xi}} L_{\Omega}$. Let ${ }_{\left(A_{B_{\Omega}}, \Omega, C^{\Omega} L_{\Omega}\right)}$ Mod be the category of left $\left(A_{B_{\Omega}}, \Omega, C^{\Omega} L_{\Omega}\right)$ modules where the three actions intertwine in a way which we now make precise.

We define a left $\left(A_{B_{\Omega}}, \Omega, C^{\Omega} L_{\Omega}\right)$-module to be a differential graded left $\Omega$ module $\zeta$ together with a differential graded left $\left(A_{B_{\Omega}}, C^{\Omega} L_{\Omega}\right)$-module structure on the space $\Gamma(\zeta)$ of sections of the underlying differential graded vector bundle $\zeta: E \rightarrow B_{\Omega}$; the two structures are required to be related by (i) and (ii) below:

(i) The left $\left(A_{B_{\Omega}}, L_{\Omega}\right)$-module structure on $\Gamma(\zeta)$ induced from the left $\Omega$-structure coincides with the restriction to $\left(A_{B_{\Omega}}, L_{\Omega}\right)$ of the given left $\left(A_{B_{\Omega}}, C^{\Omega} L_{\Omega}\right)$-module structure.

(ii) Relative to the decomposition

$$
C^{\Omega} L_{\Omega}=(s \mathfrak{g}(\xi)) \rtimes L_{\Omega},
$$

the action of the graded $A_{B_{\Omega}}$-Lie algebra $s \mathfrak{g}(\xi)$ on $\Gamma(\zeta)$ is compatible with the left $\Omega$-module structure.

We will now explain the compatibility constraint (ii). We refer to a bundle automorphism of $\xi$ which induces the identity on the base as a gauge transformation. 
Let $\mathcal{G}(\xi)$ be the group of gauge transformations of $\xi$. We identify $\mathcal{G}(\xi)$ with the group $\operatorname{Map}_{G}(P, G)$ of $G$-equivariant maps where $G$ acts on itself from the right by conjugation, the group structure being given by pointwise composition. The identification is made explicit by the association

$$
\operatorname{Map}_{G}(P, G) \longrightarrow \mathcal{G}(\xi), \quad \varphi \longmapsto \Phi: P \longrightarrow P, \quad \Phi(q)=q \varphi(q), \quad q \in P .
$$

Thus $\mathcal{G}(\xi)$ arises as the space of sections of the associated fiber bundle

$$
C(\xi): P \times_{C} G \longrightarrow B_{\Omega}
$$

where $C: G \times G \rightarrow G$ is the conjugation action $(a, b) \mapsto a b a^{-1}$. In the language of bisections, $\mathcal{G}(\xi)$ is the group of bisections of $\Omega$ which take values in the inner automorphism group bundle $C(\xi)$, cf. Example 1.4.7 on p. 25 of [31]. In particular, a vector field on $P$ whose flow generates a 1-parameter subgroup of $\mathcal{G}(\xi)$ is well known to be identifiable with a section of the adjoint bundle $\operatorname{ad}(\xi)$ on $B_{\Omega}$. In this sense, the Lie algebra $\Gamma(\operatorname{ad}(\xi))$ is the Lie algebra of the group $\mathcal{G}(\xi)$.

The group $\mathcal{G}(\xi)$ acts from the left on any vector bundle $\theta: P \times_{G} V \rightarrow B_{\Omega}$ associated with $\xi$ and the left $G$-representation $V$ in an obvious manner. In the case where the base of $\xi$ is a point, this action reduces to the left $G$-structure on $V$. For general $\xi$, the naturally induced $\mathcal{G}(\xi)$-action

$$
C^{\infty}(P, V)^{G} \times \mathcal{G}(\xi) \longrightarrow C^{\infty}(P, V)^{G}
$$

on the space $\Gamma(\theta) \cong C^{\infty}(P, V)^{G}$ of sections of $\theta$ is from the right and given by the assignment to $(\beta, \alpha) \in C^{\infty}(P, V)^{G} \times \mathcal{G}(\xi)$ of the composite $\beta \circ \alpha \in C^{\infty}(P, V)^{G}$. We will henceforth argue in terms of the associated left $\mathcal{G}(\xi)$-action

$$
\mathcal{G}(\xi) \times \Gamma(\theta) \longrightarrow \Gamma(\theta), \quad(\alpha, \beta) \longmapsto \alpha(\beta)=\beta \circ \alpha^{-1} .
$$

In the special case where the base of $\xi$ is a point, this $\mathcal{G}(\xi)$-action reduces to the left $G$-structure on $V$.

Let $\zeta$ be a differential graded left $\Omega$-module endowed as well with a left $\left(A_{B_{\Omega}}, C^{\Omega} L_{\Omega}\right)$-module structure on the space $\Gamma(\zeta)$ of sections of the underlying differential graded vector bundle $\zeta: E \rightarrow B_{\Omega}$. Relative to the decomposition (7.3), we write the action with $Y \in L_{B_{\Omega}}$ as an operation

$$
\lambda_{Y}: \Gamma(\zeta) \longrightarrow \Gamma(\zeta)
$$

of Lie derivative and the action with $v=s Y \in s \mathfrak{g}(\xi)$ as an operation

$$
i_{Y}: \Gamma(\zeta) \longrightarrow \Gamma(\zeta)
$$

of contraction. Suppose that these data satisfy the requirement (i) above. Given the section $Y$ of $\operatorname{ad}(\xi)$ (element $Y$ of $\mathfrak{g}(\xi)$ ) and the gauge transformation $\alpha$ of $\xi$ (element $\alpha$ of $\mathcal{G}(\xi)$ ), the requirement (i) implies that

$$
\alpha \lambda_{Y}=\alpha \lambda_{Y} \alpha^{-1} \alpha=\lambda_{\operatorname{Ad}_{\alpha}(Y)} \alpha: \Gamma(\zeta) \longrightarrow \Gamma(\zeta) .
$$


Accordingly, the compatibility requirement (ii) takes the form

$$
\alpha i_{Y}=i_{\operatorname{Ad}_{\alpha}(Y)} \alpha: \Gamma(\zeta) \longrightarrow \Gamma(\zeta),
$$

where $\alpha$ ranges over gauge transformations and $Y$ over sections of ad( $\xi$ ).

The cone $\left(A_{B_{\Omega}}, C^{\Omega} L_{\Omega}\right)$ on $\left(A_{B_{\Omega}}, L_{\Omega}\right)$ in the category of differential graded Lie-Rinehart algebras has an obvious underlying geometric object. Relative to the principal bundle $\xi: P \rightarrow B_{\Omega}$, consider the trivial graded vector bundle $P \times s \mathfrak{g} \rightarrow P$ whose fibers $\{q\} \times s \mathfrak{g}(q \in P)$ are concentrated in degree 1, as indicated by the notation $s \mathfrak{g}$ where $s$ refers to the suspension, and introduce the cone on $\lambda_{\Omega}$ as the differential graded Lie algebroid which, as a graded vector bundle, is the Whitney sum

$$
((\mathrm{T} P) / G) \oplus P \times_{G} s \mathfrak{g} \longrightarrow B_{\Omega}
$$

of vector bundles on $B_{\Omega}$, the Lie algebroid structure corresponding exactly to the cone $\left(A_{B_{\Omega}}, C^{\Omega} L_{\Omega}\right)$ in the category of differential graded Lie-Rinehart algebras. In particular, the anchor is induced by the composite

$$
((\mathrm{T} P) / G) \oplus P \times_{G} s \mathfrak{g} \longrightarrow(\mathrm{T} P) / G \longrightarrow \mathrm{T} B_{\Omega}
$$

of the projection to (TP)/G with the anchor of the Lie algebroid $\lambda_{\Omega}$ of $\Omega$. We refer to the resulting differential graded Lie algebroid as the cone on $\lambda_{\Omega}$ in the category of differential graded Lie algebroids.

\section{Equivariant de Rham Cohomology over a Lie Groupoid}

For intelligibility we begin by recalling the monadic description of ordinary equivariant de Rham cohomology developed in our paper [29].

Let $G$ be an ordinary Lie group, viewed as a left $G$-manifold via left translation. Given the chain complex $V$, the bicomplex $\left(\mathcal{A}^{*}\left(G, V_{*}\right), \delta, d\right)$ is defined, where $\delta$ refers to the de Rham complex operator and $d$ to the differential induced by the differential of $V$; let $\mathcal{A}(G, V)$, the $V$-valued (totalized) de Rham complex of $G$, be the chain complex arising from $\left(\mathcal{A}^{*}\left(G, V_{*}\right), \delta, d\right)$ by totalization. The notion of $(G, C \mathfrak{g})$-module and the category $\operatorname{Mod}_{(G, C \mathfrak{g})}$ of right $(G, C \mathfrak{g})$-modules have been introduced in [29]. Endow $\mathcal{A}(G, V)$ with the right $(G, C \mathfrak{g})$-module structure arising from the derivative of the $G$-action on itself and the operations of contraction and Lie derivative. See [29] for details. Let $\mathcal{C}$ be the category of real chain complexes. Consider the pair of categories

$$
(\mathcal{M}, \mathcal{D})=\left(\operatorname{Mod}_{(G, C \mathfrak{g})}, \mathcal{C}\right)
$$

and let

$$
\mathcal{G}_{(G, C \mathfrak{g})}: \mathcal{C} \longrightarrow \operatorname{Mod}_{(G, C \mathfrak{g})}
$$


be the functor which assigns to the chain complex $V$ the right $(G, C \mathfrak{g})$-module

$$
\mathcal{G}_{(G, C \mathfrak{g})} V=\mathcal{A}(G, V) .
$$

In view of an observation in [29], the functor $\mathcal{G}_{(G, C \mathfrak{g})}$ is right adjoint to the forgetful functor $\square: \operatorname{Mod}_{(G, C \mathfrak{g})} \rightarrow \mathcal{C}$ and hence defines a monad $(\mathcal{T}, u, \mu)$ over the category $\operatorname{Mod}_{(G, C \mathfrak{g})}$. In degree zero, this construction reduces to the monad spelled out in Example 2.2 above.

Let $\mathbf{V}$ be a right $(G, C \mathfrak{g})$-module. The chain complex $|\mathbf{T}(\mathbf{V})|$ arising from the dual standard construction $\mathbf{T}(\mathbf{V})$ associated with the monad $(\mathcal{T}, u, \mu)$ and the right $(G, C \mathfrak{g})$-module $\mathbf{V}$ is a resolution of $\mathbf{V}$ in the category of right $(G, C \mathfrak{g})$-modules that is injective relative to the category of chain complexes. Given a right $(G, C \mathfrak{g})$ module $\mathbf{W}$, by definition, the differential graded $\operatorname{Ext}_{((G, C \mathfrak{g}) ; \mathcal{C})}(\mathbf{W}, \mathbf{V})$ is the homology of the chain complex

$$
\operatorname{Hom}_{(G, C \mathfrak{g})}(\mathbf{W},|\mathbf{T}(\mathbf{V})|) \text {. }
$$

In particular, relative to the obvious trivial $(G, C \mathfrak{g})$-module structure on $\mathbb{R}$, the differential graded $\operatorname{Ext}_{((G, C \mathfrak{g}) ; \mathcal{C})}(\mathbb{R}, \mathbf{V})$ is the homology of the chain complex

$$
\operatorname{Hom}_{(G, C \mathfrak{g})}(\mathbb{R},|\mathbf{T}(\mathbf{V})|) \cong|\mathbf{T}(\mathbf{V})|^{(G, C \mathfrak{g})}
$$

of $(G, C \mathfrak{g})$-invariants in $|\mathbf{T}(\mathbf{V})|$.

Let $X$ be a left $G$-manifold. The de Rham complex $\mathcal{A}(X)$ acquires a right $(G, C \mathfrak{g})$-module structure in an obvious manner via the derivative of the $G$-action and the operations of contraction and Lie derivative, cf. [29]. One of the main results of [29] says that the $G$-equivariant de Rham cohomology of $X$ is canonically isomorphic to the differential $\operatorname{Ext}_{((G, C \mathfrak{g}) ; \mathcal{C})}(\mathbb{R}, \mathcal{A}(X))$. However, for reasons explained in Remark 3.1, the functor $\mathcal{G}_{(G, C \mathfrak{g})}$ does not extend to Lie groupoids since it does not even extend in degree zero, whence the monad $(\mathcal{T}, u, \mu)$ over the category $\operatorname{Mod}_{(G, C \mathfrak{g})}$ cannot extend to a monad defined suitably in terms of groupoids. The cure is provided by an extension of the monads spelled out in Examples 2.9 and 2.11 .

Thus let $\mathcal{U}: \operatorname{Mod}_{(G, C \mathfrak{g})} \longrightarrow \operatorname{Mod}_{(G, C \mathfrak{g})}$ be the functor which assigns to the right $(G, C \mathfrak{g})$-module $\mathbf{V}$ the right $(G, C \mathfrak{g})$-module $\mathcal{U}(\mathbf{V})=\mathcal{A}(G, \mathbf{V})$, the $(G, C \mathfrak{g})$-module structure being the right diagonal $(G, C \mathfrak{g})$-module structure given in [29]. This structure extends the action (2.13) coming from left translation in $G$ and the right $(G, C \mathfrak{g})$-module structure on $\mathbf{V}$. Let $\omega$ be the natural transformation given by the assignment to the right $(G, C \mathfrak{g})$-module $\mathbf{V}$ of

$$
\omega=\omega_{\mathbf{V}}: \mathbf{V} \longrightarrow \mathcal{A}^{0}(G, \mathbf{V}), \quad v \longmapsto \omega_{v}: G \rightarrow \mathbf{V}, \quad \omega_{v}(x)=v, \quad v \in \mathbf{V}, \quad x \in G,
$$

and let

$$
v: \mathcal{U}^{2} \longrightarrow \mathcal{U}
$$


be the natural transformation given by the assignment to the right $(G, C \mathfrak{g})$-module $\mathbf{V}$ of the association

$$
\nu_{\mathbf{V}}: \mathcal{A}(G, \mathcal{A}(G, \mathbf{V})) \cong \mathcal{A}(G \times G, \mathbf{V}) \longrightarrow \mathcal{A}(G, \mathbf{V})
$$

induced by the diagonal map $G \rightarrow G \times G$. The system $(\mathcal{U}, \omega, v)$ is a monad over the category $\operatorname{Mod}_{(G, C \mathfrak{g})}$. The dual standard construction $\mathbf{U}(\mathbf{V})$ associated with the monad $(\mathcal{U}, \omega, \nu)$ and the right $(G, C \mathfrak{g})$-module $\mathbf{V}$ together with $\omega_{\mathbf{V}}: \mathbf{V} \rightarrow \mathbf{U}(\mathbf{V})$ yields the resolution

$$
|\mathbf{U}(\mathbf{V})|
$$

of $\mathbf{V}$ in the category of right $(G, C \mathfrak{g})$-modules that is injective relative to the category of chain complexes and hence defines the differential $\operatorname{Ext}_{((G, C \mathfrak{g}) ; \mathcal{C})}$ as well. In particular, given the left $G$-manifold $X$, when we substitute for $\mathbf{V}$ the de Rham complex $\mathcal{A}(X)$, we again obtain the $G$-equivariant de Rham cohomology $\mathrm{H}_{G}(X)$ of $X$ as $\operatorname{Ext}_{((G, C \mathfrak{g}) ; \mathcal{C})}(\mathbb{R}, \mathcal{A}(X))$.

Guided by the construction in Example 2.11 above, we now switch from right $(G, C \mathfrak{g})$-modules, introduced in [29], to left $(G, C \mathfrak{g})$-modules. Let $\mathbf{V}$ be a chain complex, endowed with a left $G$-chain complex structure and with a left $(C \mathfrak{g})$ module structure. We write the action with $Y \in \mathfrak{g}$ as a Lie derivative $\lambda_{Y}: \mathbf{V} \rightarrow \mathbf{V}$ and the action with $s Y \in s \mathfrak{g}$ as a contraction $i_{Y}: \mathbf{V} \rightarrow \mathbf{V}$. We remind the reader that, as a graded Lie algebra, $C \mathfrak{g}=(s \mathfrak{g}) \rtimes \mathfrak{g}$; cf. the beginning of Section 5 above. The $G$-and $(C \mathfrak{g})$-module structures are said to combine to a left $(G, C \mathfrak{g})$-module structure when the left $\mathfrak{g}$-action is the derivative of the left $G$-action and when

$$
x\left(i_{Y}(v)\right)=\left(i_{\operatorname{Ad}_{x} Y}(x v)\right), \quad x \in G, \quad Y \in \mathfrak{g}, \quad v \in \mathbf{V} .
$$

We note that, when $\mathbf{V}$ is a left $(G, C \mathfrak{g})$-module, plainly

$$
x\left(\lambda_{Y}(v)\right)=\left(\lambda_{\operatorname{Ad}_{x} Y}(x v)\right), \quad x \in G, \quad Y \in \mathfrak{g}, \quad v \in \mathbf{V} .
$$

The crucial example of a left $(G, C \mathfrak{g})$-module is the de Rham complex $\mathcal{A}(Q)$ of a smooth manifold $Q$ acted upon from the right by $G$, the left $(C \mathfrak{g})$-module structure on $\mathcal{A}(Q)$ being given by the operations of contraction and Lie derivative. More generally, given the right $G$-manifold $Q$ and the left $(G, C \mathfrak{g})$-module $\mathbf{V}$, the $\mathbf{V}$-valued de Rham complex $\mathcal{A}(Q, \mathbf{V})$ acquires a diagonal left $(G, C \mathfrak{g})$-module structure. For the case of right $(G, C \mathfrak{g})$-modules rather than left $(G, C \mathfrak{g})$-modules, the diagonal structure is given in [29]. With the obvious notion of morphism, left $(G, C \mathfrak{g})$-modules constitute a category ${ }_{(G, C \mathfrak{g})}$ Mod.

We now extend the monad $(\mathcal{U}, \omega, \nu)$ over the category $\operatorname{Mod}_{(G, C \mathfrak{g})}$ reproduced above, but over the category ${ }_{(G, C \mathfrak{g})}$ Mod: Let $\xi: P \rightarrow B$ be a principal right $G$-bundle. Further, let

$$
\mathcal{U}_{\xi}:{ }_{(G, C \mathfrak{g})} \operatorname{Mod} \longrightarrow{ }_{(G, C \mathfrak{g})} \operatorname{Mod}
$$


be the functor which assigns to the left $(G, C \mathfrak{g})$-module $\mathbf{V}$ the left $(G, C \mathfrak{g})$-module

$$
\mathcal{U}_{\xi}(\mathbf{V})=\mathcal{A}(P, \mathbf{V})
$$

the $(G, C \mathfrak{g})$-module structure on $\mathcal{A}(P, \mathbf{V})$ being the left diagonal $(G, C \mathfrak{g})$-module structure coming from the right action of $G$ on $P$ and the left $(G, C \mathfrak{g})$-module structure on $\mathbf{V}$. This left diagonal $(G, C \mathfrak{g})$-module structure on $\mathcal{A}(P, \mathbf{V})$ extends the action (2.20). Let $\omega$ be the natural transformation given by the assignment to the left $(G, C \mathfrak{g})$-module $\mathbf{V}$ of

$$
\omega=\omega_{\mathbf{V}}: \mathbf{V} \longrightarrow \mathcal{A}^{0}(P, \mathbf{V}), \quad v \longmapsto \omega_{v}: P \rightarrow \mathbf{V}, \quad \omega_{v}(y)=v, \quad v \in \mathbf{V}, \quad y \in P,
$$

and let

$$
v: \mathcal{U}_{\xi}^{2} \longrightarrow \mathcal{U}_{\xi}
$$

be the natural transformation given by the assignment to the left $(G, C \mathfrak{g})$-module $\mathbf{V}$ of the association

$$
\nu_{\mathbf{V}}: \mathcal{A}(P, \mathcal{A}(P, \mathbf{V})) \cong \mathcal{A}(P \times P, \mathbf{V}) \longrightarrow \mathcal{A}(P, \mathbf{V})
$$

induced by the diagonal map $P \rightarrow P \times P$ of $P$. The system $\left(\mathcal{U}_{\xi}, \omega, v\right)$ is a monad over the category $(G, C \mathfrak{g})$ Mod. Then the dual standard construction $\mathbf{U}_{\xi}(\mathbf{V})$ associated with the monad $\left(\mathcal{U}_{\xi}, \omega, v\right)$ and the left $(G, C \mathfrak{g})$-module $\mathbf{V}$ together with $\omega_{\mathbf{V}}: \mathbf{V} \rightarrow$ $\mathbf{U}_{\xi}(\mathbf{V})$ yields the resolution

$$
\left|\mathbf{U}_{\xi}(\mathbf{V})\right|
$$

of $\mathbf{V}$ in the category of left $(G, C \mathfrak{g})$-modules that is injective relative to the category of chain complexes. Indeed, $E P / G$ is also a classifying space for $G$. More precisely, the injection of $G$ into $P$ as a specific fiber induces an injection $E G \rightarrow E P$ of right simplicial $G$-manifolds; in fact, $E G$ being a simplicial Lie group, the projection $P \rightarrow B$ induces the principal simplicial $E G$-bundle $E P \rightarrow E B$. This simplicial $E G$-bundle, in turn, induces the simplicial fiber bundle $E P / G \rightarrow E B$ having fiber $B G=E G / G$. However, since $E B$ is contractible, the injection $E G / G \rightarrow E P / G$ is a homotopy equivalence. Consequently $E P / G$ is a classifying space for $G$ as well, whence (8.6) is indeed a resolution of $\mathbf{V}$ in the category of left $(G, C \mathfrak{g})$-modules that is injective relative to the category of chain complexes.

In particular, after a choice of base point of $B$ has been made, the obvious restriction map from $\left|\mathbf{U}_{\xi}(\mathbf{V})\right|$ to $|\mathbf{U}(\mathbf{V})|$ where $|\mathbf{U}(\mathbf{V})|$ is a resolution of the kind (8.3) but constructed for left $(G, C \mathfrak{g})$-modules rather than right ones is a comparison map of resolutions. Hence the resolution (8.6) defines the differential $\operatorname{Ext}_{((G, C \mathfrak{g}) ; \mathcal{C})}$ as well. In particular, given the left $G$-manifold $X$, when we take $\mathbf{V}$ 
to be the de Rham complex $\mathcal{A}(X)$, endowed with the induced left $(G, C \mathfrak{g})$-module structure, the $G$-equivariant de Rham cohomology $\mathrm{H}_{G}(X)$ of $X$ results as the homology of

$$
\operatorname{Hom}_{(G, C \mathfrak{g})}\left(\mathbb{R},\left|\mathbf{U}_{\xi}(\mathcal{A}(X))\right|\right) .
$$

Let now $\Omega$ be a locally trivial Lie groupoid and let $f: M \rightarrow B_{\Omega}$ be a left $\Omega$-manifold; thus $f$ is endowed with a left $\Omega$-action of the kind (3.5). Our aim is to give a meaning to the $\Omega$-equivariant de Rham cohomology $\mathrm{H}_{\Omega}(f)$ of $f$. Elaborating somewhat on an observation of Seda's [38] quoted above, we shall construct a functor $\mathcal{A}(\Omega, \cdot)$ which assigns to the left $\left(A_{B_{\Omega}}, \Omega, C^{\Omega} L_{\Omega}\right)$-module $\zeta$-this is a vector bundle $\zeta: E \rightarrow B_{\Omega}$ with additional structure-a left $\left(A_{B_{\Omega}}, \Omega, C^{\Omega} L_{\Omega}\right)$-module $\mathcal{A}(\Omega, \zeta)$, similar to the vector bundle $F(\Omega, \vartheta)$ on $B_{\Omega}$ associated in Section 3 above with the left $\Omega$-module $\vartheta$; however, as a vector bundle on $B_{\Omega}$, over the object $q$ of $B_{\Omega}$, the fiber will be the de Rham complex $\mathcal{A}\left(\Omega^{q}, E_{q}\right)$ of $\Omega^{q}$ having as coefficients the fiber $E_{q}=\zeta^{-1}(q)$ rather than just the $\vartheta^{-1}(q)$-valued functions on $\Omega^{q}$ as in Example 2.11. Substituting for $\zeta$ the appropriate vector bundle arising from $f$ in a way to be made precise below, we will eventually arrive at the $\Omega$-equivariant de Rham cohomology $\mathrm{H}_{\Omega}(f)$ of $f$.

Given the left $\left(A_{B_{\Omega}}, \Omega, C^{\Omega} L_{\Omega}\right)$-module $\zeta: E \rightarrow B_{\Omega}$, let

$$
\mathcal{A}(\Omega, \zeta): \mathcal{A}(\Omega, E) \longrightarrow B_{\Omega}
$$

be the $\left(A_{B_{\Omega}}, \Omega, C^{\Omega} L_{\Omega}\right)$-module whose underlying vector bundle decomposes, for any object $q \in B_{\Omega}$, as the induced vector bundle having

$$
\Omega^{q} \times \Omega_{q}^{q} \mathcal{A}\left(\Omega^{q}, \zeta^{-1}(q)\right)
$$

as total space; thus, once a choice of $q \in B_{\Omega}$ has been made, as a vector bundle, $\mathcal{A}(\Omega, \zeta)$ amounts to the vector bundle associated with the principal right $\Omega_{q}^{q}$-bundle $t: \Omega^{q} \rightarrow B_{\Omega}$ and the left diagonal $\Omega_{q}^{q}$-representation on $\mathcal{A}\left(\Omega^{q}, \zeta^{-1}(q)\right)$ induced from the right $\Omega_{q}^{q}$-action on $\Omega^{q}$ and the left $\Omega_{q}^{q}$-action on $\zeta^{-1}(q)$. In Proposition 3.3 above, we have spelled out this kind of decomposition for the smooth functions functor $\mathcal{A}^{0}$ with appropriate values rather than for the entire de Rham functor $\mathcal{A}$. Being, as a vector bundle, associated with the principal bundle $t: \Omega^{q} \rightarrow B_{\Omega}$, the vector bundle $\mathcal{A}(\Omega, \zeta)$ acquires a left $\Omega$-module structure in the standard way, and the operations of contraction and Lie derivative relative to the fundamental vector fields coming from the $\Omega_{q}^{q}$-action turn $\mathcal{A}(\Omega, \zeta)$ into a left $\left(A_{B_{\Omega}}, \Omega, C^{\Omega} L_{\Omega}\right)$-module. This module structure extends the left diagonal $G$-structure (2.20) and the left diagonal $(G, C \mathfrak{g})$-structure on a de Rham complex $\mathcal{A}(Q, \mathbf{V})$ of a smooth right $G$-manifold $Q$ mentioned earlier.

Let $\mathcal{C}_{B_{\Omega}}$ be the category of split topological chain complexes over $B_{\Omega}$. Thus an object of $\mathcal{C}_{B_{\Omega}}$ is a differential graded topological vector bundle on $B_{\Omega}$ such that each differential, that is, operator (morphism of topological vector bundles) of the 
kind $d: \zeta_{k} \rightarrow \zeta_{k-1}$ splits (i. e. admits a retraction), and the morphisms in $\mathcal{C}_{B_{\Omega}}$ are the split morphisms of split topological chain complexes over $B_{\Omega}$.

The assignment to the left $\left(A_{B_{\Omega}}, \Omega, C^{\Omega} L_{\Omega}\right)$-module $\zeta$ of the vector bundle

$$
\mathcal{U}(\zeta)=\mathcal{A}(\Omega, \zeta): \mathcal{A}(\Omega, E) \longrightarrow B_{\Omega},
$$

cf. (8.7) above, endowed with the left $\left(A_{B_{\Omega}}, \Omega, C^{\Omega} L_{\Omega}\right)$-module structure explained above is an endofunctor

$$
\mathcal{U}:{ }_{\left(A_{B_{\Omega}}, \Omega, C^{\Omega} L_{\Omega}\right)} \operatorname{Mod} \longrightarrow{ }_{\left(A_{B_{\Omega}}, \Omega, C^{\Omega} L_{\Omega}\right)} \operatorname{Mod}
$$

on ${ }_{\left(A_{B_{0}}, \Omega, C^{\Omega} L_{\Omega}\right)}$ Mod. Likewise the construction (8.1) of the natural transformation $\omega$ and the construction (8.2) of the natural transformation $v$ extend, and we obtain a monad $(\mathcal{U}, \omega, v)$ over the category ${ }_{\left(A_{B_{\Omega}}, \Omega, C^{\Omega} L_{\Omega}\right)}$ Mod. Then the dual standard construction $\mathbf{U}(\zeta)$ associated with the monad $(\mathcal{U}, \omega, \nu)$ and the left $\left(A_{B_{\Omega}}, \Omega\right.$, $C^{\Omega} L_{\Omega}$ )-module $\zeta$, together with $\omega_{\zeta}$, yields the resolution $|\mathbf{U}(\zeta)|$ of $\zeta$ in the category of left $\left(A_{B_{\Omega}}, \Omega, C^{\Omega} L_{\Omega}\right)$-modules that is injective relative to the category $\mathcal{C}_{B_{\Omega}}$ and hence defines the differential $\operatorname{Ext}_{\left(\left(A_{B_{\Omega}}, \Omega, C^{\Omega} L_{\Omega}\right) ; \mathcal{C}_{B_{\Omega}}\right)}$. Indeed, the chain complex

$|\mathbf{U}(\zeta)|$

arising from the dual standard construction $\mathbf{U}(\zeta)$ associated with $\zeta$ together with

$$
\omega_{\zeta}: \zeta \longrightarrow|\mathbf{U}(\zeta)|
$$

is an injective resolution of $\zeta$ in the category of left $\left(A_{B_{\Omega}}, \Omega, C^{\Omega} L_{\Omega}\right)$-modules relative to the category $\mathcal{C}_{B_{\Omega}}$. Given the left $\left(A_{B_{\Omega}}, \Omega, C^{\Omega} L_{\Omega}\right)$-module $\eta$, the differential graded $\operatorname{Ext}_{\left(\left(A_{B_{\Omega}}, \Omega, C^{\Omega} L_{\Omega}\right) ; C_{B_{\Omega}}\right)}(\eta, \zeta)$ is the homology of the chain complex

$$
\operatorname{Hom}_{\left(A_{B_{\Omega}}, \Omega, C^{\Omega} L_{\Omega}\right)}(\eta,|\mathbf{U}(\zeta)|) \text {. }
$$

Similarly as in Section 3 above, we now unravel the present functor $\mathcal{U}$. Let $\xi: P \rightarrow B_{\Omega}$ be a principal right $G$-bundle whose gauge groupoid is isomorphic to $\Omega$, and suppose that the vector bundle $\zeta$ that underlies the $\left(A_{B_{\Omega}}, \Omega, C^{\Omega} L_{\Omega}\right)$-module under consideration is associated with $\xi$. Relative to $\xi$, as a topological vector bundle, $\zeta$ then amounts to an induced vector bundle of the kind

$$
P \times_{G} \mathbf{V} \longrightarrow B_{\Omega},
$$

for a suitable $(G, C \mathfrak{g})$-module $\mathbf{V}$, each constituent of the underlying graded vector space being suitably topologized. Then, relative to $\xi$, as a topological vector bundle, $\mathcal{U}(\zeta)$ amounts to an induced vector bundle of the kind

$$
P \times_{G} \mathcal{A}(G, \mathbf{V}) \longrightarrow B_{\Omega},
$$

each constituent of the $\mathbf{V}$-valued de Rham complex $\mathcal{A}(G, \mathbf{V})$ of $G$ being suitably topologized and turned into a left $(G, C \mathfrak{g})$-module via the diagonal action.

Let $\mathcal{A}_{f}: \mathcal{A}_{f}(M) \rightarrow B_{\Omega}$ be the vector bundle over $B_{\Omega}$ having as fiber over $q \in B_{\Omega}$ the ordinary de Rham complex $\left(\mathcal{A}_{f}\right)^{-1}(q)=\mathcal{A}\left(F_{q}\right)$ of the fiber $F_{q}=f^{-1}(q)$. As a topological vector bundle, once a choice of $q \in B_{\Omega}$ has been made, $\mathcal{A}_{f}$ plainly 
amounts to an induced vector bundle of the kind $\Omega^{q} \times{ }_{\Omega_{q}^{q}} \mathcal{A}\left(F_{q}\right) \longrightarrow B_{\Omega}$. The left action of $\Omega$ on $f$ induces a left $\Omega$-module structure on $\mathcal{A}_{f}$, and the operations of contraction and Lie derivative relative to the fundamental vector fields coming from the $\Omega_{q}^{q}$-action turn $\mathcal{A}_{f}$ into a left $\left(A_{B_{\Omega}}, \Omega, C^{\Omega} L_{\Omega}\right)$-module.

We now take the left $\left(A_{B_{\Omega}}, \Omega, C^{\Omega} L_{\Omega}\right)$-module $\zeta$ to be the vector bundle $\mathcal{A}_{f}$ and, furthermore, we take $\eta$ to be the trivial real line bundle on $B_{\Omega}$, endowed with the trivial left $\left(A_{B_{\Omega}}, \Omega, C^{\Omega} L_{\Omega}\right)$-module structure. Then the chain complex

$$
\operatorname{Hom}_{\left(A_{B_{\Omega}}, \Omega, C^{\Omega} L_{\Omega}\right)}(\eta,|\mathbf{U}(\zeta)|)
$$

defines the relative $\operatorname{Ext}_{\left(\left(A_{B_{\Omega}}, \Omega, C^{\Omega} L_{\Omega}\right) ; \mathcal{C}_{B_{\Omega}}\right)}(\eta, \zeta)$ which, in turn, we take as the definition of the $\Omega$-equivariant de Rham cohomology $\mathrm{H}_{\Omega}(f)$ of $f$.

THEOREM 8.1. For any object $q \in B_{\Omega}$, restriction induces an isomorphism of the $\Omega$-equivariant de Rham cohomology $\mathrm{H}_{\Omega}(f)$ of $f$ onto the ordinary $\Omega_{q}^{q}$-equivariant de Rham cohomology $\mathrm{H}_{\Omega_{q}^{q}}\left(F_{q}\right)$ of the fiber $F_{q}=f^{-1}(q)$.

Proof. The argument follows the pattern of the proof of Proposition 3.6.

Indeed, as noted above, the differential graded vector bundle $\mathcal{U}(\zeta)$ reduces to an object of the kind (8.11). Hence, given the trivial $\left(A, \Omega, C^{\Omega} L_{\Omega}\right)$-module $\eta$ having as underlying vector bundle on $B_{\Omega}$ the trivial real line bundle, the chain complex

$$
\operatorname{Hom}_{\left(A_{B_{\Omega}}, \Omega, C^{\Omega} L_{\Omega}\right)}(\eta,|\mathbf{U}(\zeta)|)
$$

reduces to the chain complex

$$
\operatorname{Hom}_{(G, C \mathfrak{g})}\left(\mathbb{R},\left|\mathbf{U}_{\xi}(\mathbf{V})\right|\right)
$$

which, in turn, as noted above, computes the relative $\operatorname{Ext}_{((G, C \mathfrak{g}) ; \mathcal{C})}(\mathbb{R}, \mathbf{V})$. Taking $G$ to be a vertex group, we may suppose that $f$ is the fiber bundle associated with $\xi$ and the left $G$-manifold $F$. Substituting $\mathcal{A}(F)$ for $\mathbf{V}$ and applying one of the main results of [29], we conclude that the $\Omega$-equivariant de Rham cohomology $\mathrm{H}_{\Omega}(f)$ of $f$ reduces to the ordinary $G$-equivariant de Rham cohomology $\mathrm{H}_{G}(F)$ of $F$.

\section{Comparison with Other Notions of Lie Groupoid Cohomology}

A possible notion of groupoid cohomology is given by the ordinary singular cohomology of the geometric realization of the nerve of the groupoid under consideration. This kind of cohomology is explored in e.g., $[9,43]$ and elsewhere. Recall that any stack admits a groupoid presentation and that the singular cohomology of a stack presented by a groupoid is defined to be the cohomology of the nerve of the groupoid. Likewise, given a differentiable stack presented by the Lie groupoid $\Lambda$, the nerve $\mathcal{N}(\Lambda)$ of $\Lambda$ is a simplicial manifold (for $p \geq 0$, we will denote the 
homogeneous degree $p$ constituent by $\Lambda_{p}$ ), and the de Rham cohomology of the stack is defined to be the total cohomology of the cosimplicial de Rham complex

$$
\left(\mathcal{A}^{p, q}(\Lambda), \delta, \varepsilon, \eta\right)_{p \geq 0, q \geq 0}
$$

here $\mathcal{A}^{p, q}(\Lambda)=\mathcal{A}^{q}\left(\Lambda_{p}\right)$, the cosimplicial operators $\varepsilon$ and $\eta$ are induced by the ordinary simplicial operators in the standard manner by dualization and, for $p, q \geq 0$,

$$
\delta: \mathcal{A}^{q}\left(\Lambda_{p}\right) \rightarrow \mathcal{A}^{q+1}\left(\Lambda_{p}\right)
$$

is the ordinary de Rham operator. See e.g., [4] and the literature there. We will refer to this cohomology as the stack de Rham cohomology of $\Lambda$. As already noted in the introduction, when the underlying Lie groupoid $\Lambda$ is actually an ordinary Lie group, viewed as a Lie groupoid with a single object, this cosimplicial de Rham complex amounts to the construction developed and explored by Bott et al. $[5,6,11,40]$.

Let $\Omega$ be a Lie groupoid and $f: M \rightarrow B_{\Omega}$ a left $\Omega$-manifold, cf. Section 3 above; thus $f$ is endowed with a left $\Omega$-action given by a commutative diagram of the kind (3.5) such that the obvious associativity constraint is satisfied. Let $\Lambda=\Omega \ltimes M$ be the associated action groupoid. As noted above, in the literature, the cohomology of the associated stack is defined as the singular cohomology of the geometric realization $B(\Omega \ltimes M)$ of the nerve of $\Omega \ltimes M$. In the case, where the Lie groupoid under discussion is a Lie group, that geometric realization reduces to the ordinary homotopy quotient. While the de Rham functor does not apply to $B(\Omega \ltimes M)$ directly, the definition in terms of the total cohomology of the associated cosimplicial de Rham complex yields a notion of de Rham cohomology for $\Omega \ltimes M$, the stack de Rham cohomology of $\Omega \ltimes M$.

More reprecisely, we will write the nerve of $\Omega \ltimes M$ as $\mathcal{N}(\Omega, f)$. The target map $t$ yields the simplicial manifold

$$
t: \mathcal{N}(\Omega, f) \longrightarrow B_{\Omega}
$$

over $B_{\Omega}$ where the notation $t$ is slightly abused. The simplicial manifold (9.2) over $B_{\Omega}$ can be seen as the standard construction associated with the corresponding comonad that is lurking behind. The cosimplicial de Rham complex (9.1) now takes the form

$$
\left(\mathcal{A}^{p, q}(\Omega, f), \delta, \varepsilon, \eta\right)_{p \geq 0, q \geq 0}
$$

here $\mathcal{A}^{p, q}(\Omega, f)=\mathcal{A}^{q}\left(\mathcal{N}(\Omega, f)_{p}\right)$, the cosimplicial operators $\varepsilon$ and $\eta$ are induced by the ordinary simplicial operators in the standard manner by dualization, and

$$
\delta: \mathcal{A}^{q}\left(\mathcal{N}(\Omega, f)_{p}\right) \longrightarrow \mathcal{A}^{q+1}\left(\mathcal{N}(\Omega, f)_{p}\right)
$$

is the ordinary de Rham operator. The target map $t: \Omega \rightarrow B_{\Omega}$ endows Id: $B_{\Omega} \rightarrow$ $B_{\Omega}$ with a left $\Omega$-structure, the trivial structure. For the special case where $f=$ 
Id: $B_{\Omega} \rightarrow B_{\Omega}$, a de Rham complex of the kind (9.3) is, indeed, suggested in Section 2 of [6] as a possible definition for the cohomology of the Haefliger groupoid arising in Haefliger's classification theory for foliations but, beware, no such suggestion is made in [6] concerning equivariant cohomology over that groupoid.

To relate the above constructions to the approach to equivariant cohomology over a groupoid developed in the present paper, suppose now that $\Omega$ is locally trivial. Let $\mathcal{A}_{f}: \mathcal{A}_{f}(M) \rightarrow B_{\Omega}$ be the differential graded vector bundle over $B_{\Omega}$ having as fiber over $q \in B_{\Omega}$ the ordinary de Rham complex $\mathcal{A}_{f}^{-1}(q)=\mathcal{A}\left(F_{q}\right)$ of the fiber $F_{q}=f^{-1}(q)$. The construction developed in the present paper starts from a differential graded vector bundle of the kind

$$
\mathcal{A}\left(\Omega, \mathcal{A}_{f}\right): \mathcal{A}\left(\Omega, \mathcal{A}_{f}(M)\right) \longrightarrow B_{\Omega}
$$

having as fiber over $q \in B_{\Omega}$ the ordinary de Rham complex

$$
\left(\mathcal{A}\left(\Omega, \mathcal{A}_{f}\right)\right)^{-1}(q)=\mathcal{A}\left(\Omega^{q}, \mathcal{A}\left(F_{q}\right)\right)
$$

of $\mathcal{A}\left(F_{q}\right)$-valued forms on the manifold $\Omega^{q}$ of all morphisms $u$ in $\Omega$ having $q$ as their source, i.e., $s(u)=q$. This is the first step of the construction, and iterating the construction yields a cosimplicial differential graded vector bundle $\mathbf{U}\left(\mathcal{A}_{f}\right)$ over $B_{\Omega}$. Let $\eta$ be the trivial line bundle on $B_{\Omega}$, endowed with the trivial $\Omega$-structure. The cohomology is then that given by a complex of the kind

$$
\operatorname{HOM}\left(\eta,\left|\mathbf{U}\left(\mathcal{A}_{f}\right)\right|\right)
$$

where the notation HOM refers to the requisite structure present in the construction and not spelled out here and where the notation $\left|\mathbf{U}\left(\mathcal{A}_{f}\right)\right|$ refers to the chain complex which results from totalization.

The chain complex arising from the cosimplicial de Rham complex (9.3) is substantially different from the chain complex (9.5) developed in the present paper, and the two constructions are not isomorphic, perhaps not even related in an obvious manner, unless the groupoid $\Omega$ is a group. Suffice it to point out here that the operation of applying the functor of taking the space of sections to the construction in the present paper yields an object which formally looks somewhat like the cosimplicial de Rham complex (9.3). Indeed, a first step towards unveiling the connections between the two construction consists, perhaps, in this observation: given the fiber bundle $\phi: E \rightarrow B$, let $C_{\phi}^{\infty}$ be the vector bundle on $B$ having as fiber over $b \in B$ the algebra $C^{\infty}\left(F_{b}\right)$ of smooth functions on the fiber $F_{b}$; then, as a $C^{\infty}(B)$ module, the space of sections of $C_{\phi}^{\infty}$ yields the smooth functions on the total space $E$.

The cosimplicial de Rham complex (9.3) does not arise by the operation of iterating the construction of a relatively injective module with respect to the groupoid $\Omega$, though, unless $\Omega$ is a group and hence does not yield a kind of injective resolution, and a suitable monad leading to that kind of cohomology is not in sight since one cannot naively dualize the comonad lurking behind the simplicial object 
(9.2) as one does in the group case. Indeed, in the group case, in a sense, the Hochschild-Mostow injective resolution arises from application of the functor $C^{\infty}$ to the corresponding simplicial object, but such a claim cannot be made over a general Lie groupoid and the difficulty was overcome only in [32] where the requisite (relatively) injective resolution was exhibited. Working out the exact features of that kind of construction and of the precise relationship thereof with the construction (9.5) is an interesting endeavor in itself. However, as noted already in the introduction, the notions of injective module and injective resolution are fundamental. They have been developed by the masters to cope with situations where projective modules are not available. Without the notion of injective module there would be no sheaf cohomology, for example.

One can show in a roundabout way, however, that the stack de Rham cohomology of $\Omega \ltimes M$ coincides with the $\Omega$-equivariant de Rham cohomology $\mathrm{H}_{\Omega}(f)$ of $f$ developed in the present paper. Indeed, pick a base point $q \in B_{\Omega}$; by Theorem 8.1, restriction induces an isomorphism of $\mathrm{H}_{\Omega}(f)$ onto the ordinary $\Omega_{q}^{q}$-equivariant de Rham cohomology $\mathrm{H}_{\Omega_{q}^{q}}\left(F_{q}\right)$ of the fiber $F_{q}=f^{-1}(q)$. Since $\Omega$ is locally trivial, by Morita equivalence, cf. e.g., [36], the action groupoid $\Omega \ltimes M$ is equivalent to the action groupoid $\Omega_{q}^{q} \ltimes F_{q}$, restriction induces an isomorphism from the stack de Rham cohomology of $\Omega \ltimes M$ onto the stack de Rham cohomology of $\Omega_{q}^{q} \ltimes F_{q}$, and the stack de Rham cohomology of $\Omega_{q}^{q} \ltimes F_{q}$ amounts to the ordinary $\Omega_{q}^{q}$-equivariant cohomology $\mathrm{H}_{\Omega_{q}^{q}}\left(F_{q}\right)$ of $F_{q}$. Putting the various isomorphisms together we conclude that the stack de Rham cohomology of $\Omega \ltimes M$ coincides with the $\Omega$-equivariant de Rham cohomology $\mathrm{H}_{\Omega}(f)$ of $f$. Thus Theorem 8.1 implies that our notion of $\Omega$-equivariant de Rham cohomology $\mathrm{H}_{\Omega}(f)$ of $f$ yields a description of the stack de Rham cohomology of $\Omega \ltimes M$ as a relative derived functor. This kind of reasoning also reveals that the real singular cohomology of an action groupoid of the kind $\Omega \ltimes M$ (defined as the ordinary singular cohomology of the geometric realization $B(\Omega \ltimes M)$ of the nerve of $\Omega \ltimes M)$ coincides with the equivariant de Rham cohomology $\mathrm{H}_{\Omega}(f)$ of $f$. Furthermore, this reasoning also implies in a roundabout manner that, suitably interpreted, the equivariant de Rham cohomology of a locally trivial Lie groupoid satisfies Morita equivalence.

\section{Outlook}

Various questions remain open including the following ones:

1. Does the extension of Bott's decomposition lemma given in [29] still generalize to the present situation in the sense that the functor $\mathcal{U}$ given as (8.9) induces a decomposition of the corresponding standard construction into the standard construction defining the ordinary Lie groupoid cohomology, spelled out above as Theorem 3.5, and the standard construction defining the equivariant Lie algebroid cohomology that corresponds to the theory developed for an 
extension of Lie-Rinehart algebras in Section 6? If the answer to this question is yes, what does this extension of the decomposition lemma then signify?

2. Is there a corresponding Chern-Weil construction and, if so, what does it signify? More precisely: Let $G$ be a Lie group and $\mathfrak{g}$ its Lie algebra. Filtering the ordinary bar-de Rham bicomplex of $G$ by the complementary degree we obtain a spectral sequence $\left(\mathrm{E}_{r}, d_{r}\right)$ explored by Bott in his seminal paper [5]. Bott has in particular shown that this spectral sequence has

$$
\mathrm{E}_{1}=\mathrm{H}_{\text {cont }}^{*}\left(G, \mathrm{~S}\left[\left(s^{2} \mathfrak{g}\right)^{*}\right]\right)
$$

where $\mathrm{H}_{\text {cont }}^{*}$ refers to continuous cohomology and where $\mathrm{S}\left[\left(s^{2} \mathfrak{g}\right)^{*}\right]$ denotes the symmetric algebra on the dual of the double suspension $s^{2} \mathfrak{g}$ of $\mathfrak{g}$. Let now $X$ be a left $G$-manifold and use the notation $\mathrm{S}^{\mathrm{c}}$ for the symmetric coalgebra functor. In [29] we have extended that spectral sequence to a spectral sequence $\left(\mathrm{E}_{r}, d_{r}\right)$, referred to in [29] as the generalized Bott spectral sequence, having

$$
\mathrm{E}_{1}=\mathrm{H}_{\text {cont }}^{*}\left(G, \operatorname{Hom}\left(\mathrm{S}^{\mathrm{c}}\left[s^{2} \mathfrak{g}\right], \mathcal{A}(X)\right)\right) .
$$

In particular when $G$ is compact, $\mathrm{H}_{\text {cont }}^{*}\left(G, \operatorname{Hom}\left(\mathrm{S}^{\mathrm{c}}\left[s^{2} \mathfrak{g}\right], \mathcal{A}(X)\right)\right)$ reduces to the $G$-invariants $\operatorname{Hom}\left(\mathrm{S}^{\mathrm{c}}\left[s^{2} \mathfrak{g}\right], \mathcal{A}(X)\right)^{G}$. But the elements of the latter are precisely homogeneous $G$-invariant $\mathcal{A}(X)$-valued polynomial maps on (the double suspension of) $\mathfrak{g}$ and these maps, in turn, are precisely the Chern-Weil maps. Thus the spectral sequence $\left(\mathrm{E}_{r}, d_{r}\right)$ explains the classical Chern-Weil construction. In [29], we have gone further: One of the results of that paper spells out the standard complex calculating the $G$-equivariant cohomology of $X$ as a perturbation of the ordinary bar complex operator. This raises the question of the extent to which these facts carry over to general Lie groupoids. In [24], we have worked out a preliminary step at the infinitesimal level.

3. How do the notions of duality and modular class developed in [25] extend to groupoids?

4. Is there a quasi-version of the theory of the kind developed at the infinitesimal level in [27] and, if so, what does it signify? Is this theory related with the issue spelled out in the previous item?

5. The functor $\operatorname{Ext}_{\left(\left(A_{B_{\Omega}}, \Omega, C^{\Omega} L_{\Omega}\right) ; \mathcal{C}_{B_{\Omega}}\right)}$ defined in Section 8 can also be viewed as a functor of the kind $\operatorname{Ext}_{\left(\left(A_{B_{\Omega}}, \mathcal{G}, C^{\Omega} L_{\Omega}\right) ; \mathcal{C}_{B_{\Omega}}\right)}$ where $\mathcal{G}$ is a suitable group of bisections of $\Omega$. This kind of relative derived functor can presumably be constructed for general Lie-Rinehart algebras.

6. Do the van Est spectral sequences generalize to Lie groupoids? We recall briefly the situation for Lie groups. Let $G$ be a connected Lie group with Lie algebra $\mathfrak{g}$, and let $V$ be a $G$-representation. The first van Est spectral sequence $\left(E_{r}, d_{r}\right)$ associated with the data has $E_{2}$ isomorphic to $\mathrm{H}_{\mathrm{diff}}^{*}\left(G, \mathrm{H}_{\text {top }}^{*}(G, V)\right)$ and converges to the Lie algebra cohomology $\mathrm{H}^{*}(\mathfrak{g}, V)$. Here the constituent $\mathrm{H}_{\text {top }}^{*}(G, V)$ can be replaced by the ordinary $V$-valued cohomology $\mathrm{H}_{\text {top }}^{*}(K, V)$ of a maximal compact subgroup $K$ of $G$. The second van Est spectral 
sequence $\left(E_{r}, d_{r}\right)$ is associated with these data together with a choice $K$ of compact subgroup $K$ of $G$, not necessarily maximal; the spectral sequence has $E_{2}$ isomorphic to $\mathrm{H}_{\text {diff }}^{*}\left(G, \mathrm{H}_{\text {top }}^{*}(G / K, V)\right)$ and converges to the relative Lie algebra cohomology $\mathrm{H}^{*}(\mathfrak{g}, \mathfrak{k} ; V)$ where $\mathfrak{k}$ is the Lie algebra of $K$. In particular, when $K$ is a maximal compact subgroup, the homogeneous space $G / K$ is affine and hence contractible, and the spectral sequence amounts to an isomorphism $\mathrm{H}_{\text {diff }}^{*}(G, V) \cong \mathrm{H}^{*}(\mathfrak{g}, \mathfrak{k} ; V)$, referred to usually as the van Est isomorphism. This isomorphism then yields a description of an edge map in the first van Est spectral sequence. Which of these facts carry over to Lie groupoids and Lie algebroids is an interesting question. In order to treat these questions one must first define the requisite notions, for example relative Lie algebroid cohomology. A formally appropriate way to do this might consist in developing the requisite relative homological algebra, that is, the appropriate monad.

7. What is the most appropriate form of Morita equivalence relative to the notion of equivariant cohomology with respect to a locally trivial Lie groupoid? At the end of the previous section, we identified the equivariant cohomology developed in the present paper with the corresponding stack de Rham cohomology in a roundabout manner. Is there a more formal way to explain the identification and to explain why the two theories are equivalent?

8. Can equivariant de Rham theory be developed as a relative derived functor for Lie groupoids that are not locally trivial, e.g., for groupoids of the kind associated with a general foliation which is not a fiber bundle? What does the equivariant de Rham cohomology signify in this case? Presumably it would then be better to rework the approach in the language of sheaves, the present approach being the special case where the requisite sheaves are fine. Is there a description of the stack de Rham cohomology over a Lie groupoid that is not locally trivial as a relative derived functor? If the answer to both questions is yes, are the two theories the same or do they differ?

We hope to return to these issues elsewhere.

\section{References}

1. Almeida, R., Kumpera, A.: Structure produit dans la catégorie des algébroïdes de Lie. An. Acad. Brasil. Cienc. 53, 247-250 (1981)

2. Almeida, R., Molino, P.: Suites d'Atiyah et feuilletages transversalement complets. C. R. Acad. Sci. Paris I 300, 13-15 (1985)

3. Atiyah, M.F.: Complex analytic connections in fibre bundles. Trans. Am. Math. Soc. 85, 181-207 (1957)

4. Behrend, K.: On the de Rham cohomology of differentiable and algebraic stacks. Adv. Math. 198, 583-622 (2005). Math/0410255[math.AG]

5. Bott, R.: On the Chern-Weil homomorphism and the continuous cohomology of Lie groups. Adv. Math. 11, 289-303 (1973)

6. Bott, R., Shulman, H., Stasheff, J.: On the de Rham theory of certain classifying spaces. Adv. Math. 20, 43-56 (1976) 
7. Bruzzo, U., Chirio, L., Rossi, P., Rubtsov, V.N.: Equivariant cohomology and localization for Lie algebroids. Funct. Anal. Appl. 43, 18-296 (2009). Math/0506392[math.DG]

8. Cannas de Silva, A., Weinstein, A.: Lectures on Geometric Models for Noncommutative Algebras. Berkeley Mathematics Lecture Notes, vol. 10. American Mathematical Society, Providence (1999)

9. Crainic, M.: Differentiable and algebroid cohomology, van Est isomorphisms and characterstic classes. Comm. Math. Helv. 78, 681-721 (2003). Math/0008064[math.DG]

10. Dold, A., Puppe, D.: Homologie nicht-additiver Funktoren. Anwendungen. Ann. Inst. Fourier 11, 201-313 (1961)

11. Dupont, J.L.: Simplicial de Rham cohomology and characteristic classes of flat bundles. Topology 15, 233-245 (1976)

12. Duskin, J.: Simplicial methods and the interpretation of "triple" cohomology. Mem. Am. Math. Soc. 163 (1975)

13. Godement, R.: Topologie algébrique et théorie des faisceaux. Hermann, Paris (1958)

14. Higgins, P.J.: Categories and Groupoids. Van Nostrand, Princeton (1971)

15. Higgins, P.J., Mackenzie, K.: Algebraic constructions in the category of Lie algebroids. J. Algebra 129, 194-230 (1990)

16. Higgins, P.J., Mackenzie, K.: Duality for base-changing morphisms of vector bundles, modules, Lie algebroids and Poisson structures. Math. Proc. Camb. Philos. Soc. 114, 471-488 (1993)

17. Hilton, P.J., Stammbach, U.: A Course in Homological Algebra. Graduate Texts in Mathematics, vol. 4. Springer, Berlin (1971)

18. Hochschild, G.: Relative homological algebra. Trans. Am. Math. Soc. 82, 246-269 (1956)

19. Hochschild, G., Mostow, G.D.: Cohomology of Lie groups. Ill. J. Math. 6, 367-401 (1962)

20. Huebschmann, J.: Poisson cohomology and quantization. J. Reine Angew. Math. 408, 57-113 (1990)

21. Huebschmann, J.: On the quantization of Poisson algebras. In: Donato, P., Duval, C., Elhadad, J., Tuynman, G.M. (eds.) Symplectic Geometry and Mathematical Physics, Actes du colloque en l'honneur de Jean-Marie Souriau. Progress in Mathematics, vol. 99, pp. 204-233. Birkhäuser, Boston (1991)

22. Huebschmann, J.: Lie-Rinehart algebras, descent, and quantization. Fields Inst. Commun. 43, 295-316 (2004). Math.SG/0303016

23. Huebschmann, J.: Lie-Rinehart algebras, Gerstenhaber algebras, and BatalinVilkovisky algebras. Ann. Inst. Fourier 48, 425-440 (1998). Math.DG/9704005

24. Huebschmann, J.: Extensions of Lie-Rinehart algebras and the Chern-Weil construction. In: Festschrift in honour of Jim Stasheff's 60'th anniversary. Contemporary Mathematics, vol. 227, pp. 145-176 (1999). Math.DG/9706002

25. Huebschmann, J.: Duality for Lie-Rinehart algebras and the modular class. J. Reine Angew. Math. 510, 103-159 (1999). Math.DG/9702008

26. Huebschmann, J.: Differential Batalin-Vilkovisky algebras arising from twilled Lie-Rinehart algebras. Banach Cent. Publ. 51, 87-102 (2002)

27. Huebschmann, J.: Higher homotopies and Maurer-Cartan algebras: quasi-Lie-Rinehart, Gerstenhaber-, and Batalin-Vilkovisky algebras. In: Marsden, J., Ratiu, T. (eds.) The Breadth of Symplectic and Poisson Geometry, Festschrift in Honor of Alan Weinstein. Progress in Mathematics, vol. 232, pp. 237-302. Birkhäuser, Boston (2004). math.DG/0311294

28. Huebschmann, J.: Homological perturbations, equivariant cohomology, and Koszul duality. Doc. Math. (2009, to appear). Math.AT/0401160 
29. Huebschmann, J.: Relative homological algebra, homological perturbations, equivariant de Rham theory, and Koszul duality. Math.AG/0401161

30. Mackenzie, K.C.H.: Lie groupoids and Lie algebroids in differential geometry. London Mathematical Society. Lecture Note Series, vol. 124. Cambridge University Press, Cambridge (1987)

31. Mackenzie, K.C.H.: General theory of Lie groupoids and Lie algebroids. London Mathematical Society. Lecture Note Series, vol. 213. Cambridge University Press, Cambridge (2005)

32. Mackenzie, K.A.: Rigid cohomology of topological groupoids. J. Aust. Math. Soc. 26, 277-301 (1978)

33. Mac Lane, S.: Homologie des anneaux et des modules. In: Colloque de topologie algébrique, Louvain, pp. 55-80 (1956)

34. MacLane, S.: Homology. Die Grundlehren der mathematischen Wissenschaften, vol. 114. Springer, Berlin (1967)

35. Mac Lane, S.: Categories for the Working Mathematician. Graduate Texts in Mathematics, 2nd edn, vol. 5. Springer, New York (1998)

36. Moerdijk, I., Mrčun, J.: Introduction to Foliations and Lie Groupoids. Cambridge Studies in Advanced Mathematics, vol. 91. Cambridge University Press, Cambridge (2003)

37. Rinehart, G.: Differential forms for general commutative algebras. Trans. Am. Math. Soc. 108, 195-222 (1963)

38. Seda, A.K.: An extension theorem for transformation groupoids. Proc. R. Irish Acad. 75A, 255-262 (1975)

39. Segal, G.B.: Classifying spaces and spectral sequences. Publ. Math. IHES 34, 105-112 (1968)

40. Shulman, H.B.: Characteristic Classes and Foliations. Ph. D. Thesis, University of California (1972)

41. Stasheff, J.D.: Continuous cohomology of groups and classifying spaces. Bull. Am. Math. Soc. 84, 513-530 (1978)

42. Van Est, W.T.: Algèbres de Maurer-Cartan et holonomie. Ann. Fac. Sci. Toulouse Math. 5(Suppl), 93-134 (1989)

43. Weinstein, A., Xu, P.: Extensions of symplectic groupoids and quantization. J. Reine Angew. Math. 417, 159-189 (1991) 